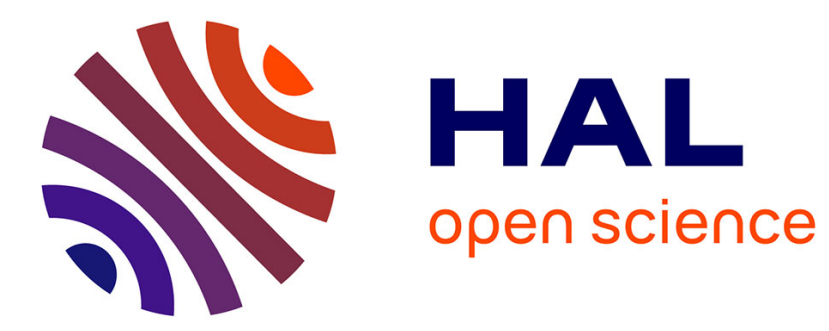

\title{
Newton polygons for character sums and Poincaré series Régis Blache
}

\section{To cite this version:}

Régis Blache. Newton polygons for character sums and Poincaré series. 2011. hal-00551461

\section{HAL Id: hal-00551461 \\ https://hal.science/hal-00551461}

Preprint submitted on 3 Jan 2011

HAL is a multi-disciplinary open access archive for the deposit and dissemination of scientific research documents, whether they are published or not. The documents may come from teaching and research institutions in France or abroad, or from public or private research centers.
L'archive ouverte pluridisciplinaire HAL, est destinée au dépôt et à la diffusion de documents scientifiques de niveau recherche, publiés ou non, émanant des établissements d'enseignement et de recherche français ou étrangers, des laboratoires publics ou privés. 


\title{
NEWTON POLYGONS FOR CHARACTER SUMS AND POINCARÉ SERIES.
}

\author{
RÉGIS BLACHE
}

\begin{abstract}
In this paper, we precise the asymptotic behaviour of Newton polygons of $L$-functions associated to character sums, coming from certain $n$ variable Laurent polynomials. In order to do this, we use the free sum on convex polytopes. This operation allows the determination of the limit of generic Newton polygons for the sum $\Delta=\Delta_{1} \oplus \Delta_{2}$ when we know the limit of generic Newton polygons for each factor. To our knowledge, these are the first results concerning the asymptotic behaviour of Newton polygons for multivariable polynomials when the generic Newton polygon differs from the combinatorial (Hodge) polygon associated to the polyhedron.
\end{abstract}

\section{INTRODUCTION}

In the following, we note $k$ a finite field with $q=p^{a}$ elements, and $k_{r}$ its degree $r$ extension in an algebraic closure $\bar{k}$ fixed once and for all. Let $\mathbf{x}=\left(x_{1}, \ldots, x_{n}\right)$ be an $n$-tuple of indeterminates, and $f(\mathbf{x})=\sum_{\mathbf{i} \in \mathbb{Z}^{n}} a_{\mathbf{i}} \mathbf{x}^{\mathbf{i}} \in k\left[\mathbf{x}, \mathbf{x}^{-1}\right]$ be a Laurent polynomial in $n$ variables with coefficients in $k$. If $\psi$ denotes a non trivial additive character of $k$, let $\psi_{r}:=\psi \circ \operatorname{Tr}_{k_{r} / k}$ be the character induced by $\psi$ on $k_{r}$; let $\chi$ be a multiplicative character of $\left(k^{\times}\right)^{n}$, and $\chi_{r}:=\chi \circ \mathrm{N}_{k_{r} / k}$ its extension to $\left(k_{r}^{\times}\right)^{n}$. From $f, \psi$ and $\chi$, we form the character sums over each extension of $k$

$$
S_{r}(f, \chi)=\sum_{\mathbf{x} \in \mathbb{G}_{m}^{n}\left(k_{r}\right)} \psi(f(\mathbf{x})) \chi(\mathbf{x}),
$$

then from these sums we define the $L$-function

$$
L(f, \chi ; T)=\exp \left(\sum_{r \geq 1} S_{r}(f, \chi) \frac{T^{r}}{r}\right) .
$$

When $\chi$ is trivial, we simply denote this function by $L(f ; T)$. From the works of Dwork and Grothendieck ( $c f .8$, [10]) we know that it is rational.

Let us begin with a trivial $\chi$; this is the most classical case in the literature. The first result about the $L$-function is due to Deligne [6, Théorème 8.4]. For a polynomial of degree $d$ prime to $p$, whose higher degree form defines a nonsingular hypersurface in the projective space $\mathbb{P}^{n-1}$, the function $L^{\prime}(f, T)$ (here the sums are defined over $\mathbb{A}^{n}$, not over $\left.\mathbb{G}_{m}^{n}\right)$ has degree $(d-1)^{n}$.

More generally, one can associate to the polynomial $f$ its Newton polyhedron at infinity, which is the convex polyhedron $\Delta$ defined in affine space $\mathbb{R}^{n}$ as the convex

1991 Mathematics Subject Classification. 11M38,13A02,52B20.

Key words and phrases. Character sums, $L$-functions, Newton polygons and polytopes. 
hull of the origin and the support of $f$ (that is: the set of $\mathbf{i} \in \mathbb{Z}^{n}$ such that $a_{\mathbf{i}}$ is non zero). Then when certain forms of $f$, coming from the facets of $\Delta$, are non degenerate, Adolphson and Sperber [1] have shown that the function $L(f ; T)^{(-1)^{n-1}}$ is a polynomial with degree $n ! V(\Delta)$, where $V$ is the usual volume over $\mathbb{R}^{n}$.

Let us denote $\alpha_{1}, \ldots, \alpha_{n ! V(\Delta)}$ the reciprocal roots of this polynomial. These are algebraic integers having the following properties: their complex modulus is $\left|\alpha_{i}\right|=q^{\frac{w_{i}}{2}}$, for integers $0 \leq w_{i} \leq n$. Moreover any conjugate (over $\operatorname{Gal}(\overline{\mathbb{Q}} / \mathbb{Q})$ ) of $\alpha_{i}$ have the same modulus. The distribution of the $w_{i}$ is known 7, Theorem 1.8]. On the other hand, for any prime $\ell \neq p, \alpha_{i}$ is a $\ell$-adic unit. Finally we have $\left|\alpha_{i}\right|_{p}=q^{-s_{i}}$, for some rational $s_{i}$ between 0 and $n$. In this paper we shall study these valuations.

As usual, we identify the $q$-adic valuations of the reciprocal roots of a polynomial with the slopes of the segments in its $q$-adic Newton polygon. We shall denote by $\mathrm{NP}_{q}(f)\left(\right.$ resp. $\left.\mathrm{NP}_{q}(f, \chi)\right)$ the $q$-adic Newton polygon of $L(f ; T)($ resp. $L(f, \chi ; T))$ in the following. If $\Pi$ is a convex polygon with length $l$, i.e. the graph of a continuous, piecewise linear function $\varphi$ over the interval $[0, l]$ affine over each of the intervals $[i-1, i]$ and such that $\varphi(0)=0$, we shall denote $\Pi=\left(\pi_{i}\right)_{1 \leq i \leq l}$ when the slope over the interval $[i-1, i]$ is $\pi_{i}$. If $\Pi_{1}$ and $\Pi_{2}$ are two convex polygons of length $l$, we shall write $\Pi_{1} \preceq \Pi_{2}$ when $\Pi_{1}$ lies above $\Pi_{2}$, and their endpoints coincide.

Adolphson et Sperber have given [1, Theorem 3.10] a lower bound for the Newton polygons of the functions $L(f ; T)^{(-1)^{n-1}}$ when $f$ runs over the (non degenerate) polynomials with fixed Newton polyhedron $\Delta$. This bound is usually called the Hodge polygon of the polyhedron $\Delta$, denoted $\operatorname{HP}(\Delta)$. This is an invariant depending only on $\Delta$, that we describe in the next paragraph. Remark that generally this is not the Hodge polygon of a crystal (cf. 12]), nor a geometric Hodge polygon, these families of polygons having all their slopes integral. However, it is shown in $[9$ that the procedure giving the Hodge polygon from $\Delta$ is the same that the one giving the Hodge spectrum at infinity of a complex polynomial having Newton polyhedron $\Delta$. This gives a Hodge theoretical interpretation of this polygon.

Denote by $C(\Delta):=\mathbb{R}_{+} \Delta$ the cone of $\Delta$ in $\mathbb{R}^{n}, M_{\Delta}:=C(\Delta) \cap \mathbb{Z}^{n}$ the monoid associated to this cone, and $\mathcal{A}_{\Delta}$ the algebra $k\left[\mathbf{x}^{M_{\Delta}}\right]$. One can define a map from $C(\Delta)$ to $\mathbb{R}_{+}$, the weight associated to $\Delta$, by

$$
w_{\Delta}(\mathbf{u})=\min \left\{\rho \in \mathbb{R}_{+}, \mathbf{u} \in \rho \Delta\right\} .
$$

The vertices of $\Delta$ lie in $\mathbb{Z}^{n}$, thus the image of $M_{\Delta}$ by $w_{\Delta}$ lies in $\mathbb{Q}_{+}$; more precisely there is a positive integer $D$ such that $\operatorname{Im} w_{\Delta} \subseteq \frac{1}{D} \mathbb{N}$. We shall call the least integer $D$ having this property the denominator of $\Delta$. The weight $w_{\Delta}$ turns the algebra $\mathcal{A}_{\Delta}$ into a graded algebra

$$
\mathcal{A}_{\Delta}=\oplus_{i \geq 0} \mathcal{A}_{\Delta, \frac{i}{D}}, \mathcal{A}_{\Delta, \frac{i}{D}}=\operatorname{Vect}\left\{\mathbf{x}^{\mathbf{u}}, w_{\Delta}(\mathbf{u})=\frac{i}{D}\right\}
$$

to which we associate the Poincaré series

$$
P_{\mathcal{A}_{\Delta}}(t):=\sum_{i \geq 0} \operatorname{dim} \mathcal{A}_{\Delta, \frac{i}{D}} t^{i}
$$

Kouchnirenko [14, Lemme 2.9] has shown that for non degenerate $f$, this series is actually a rational function. Precisely, the series $P_{\Delta}(t):=\left(1-t^{D}\right)^{n} P_{\mathcal{A}_{\Delta}}(t)$ is a polynomial with degree less than or equal to $n D$. If we set $P_{\Delta}(t):=\sum \ell_{i} t^{s_{i}}$, the Hodge polygon $\operatorname{HP}(\Delta)$ is the convex polyhedron starting at the origin, and formed 
by the segments of horizontal length $\ell_{i}$ and slope $\frac{s_{i}}{D}$. In the following, we shall also call this polygon the polygon coming from the Poincaré series $P_{\mathcal{A}_{\Delta}}$. With our notations, Adolphson and Sperber's result can be rewritten in the following way: for any polynomial $f$ in $k\left[\mathbf{x}, \mathbf{x}^{-1}\right]$, non degenerate with respect to its Newton polyhedron $\Delta$ we have $\mathrm{NP}_{q}(f) \preceq \operatorname{HP}(\Delta)$.

It is natural to ask how the polygons $\mathrm{NP}_{q}(f)$ behave when $f$ varies along the (nondegenerate) polynomials with fixed Newton polyhedron. Unfortunately these variations are difficult to control; explicit calculations for polynomials of little degree in one variable show that one cannot hope a complete answer to this question. To overcome this problem, we shall work with generic Newton polygons. Grothendieck's specialization theorem [12] ensures the lower bound of the polygons $\mathrm{NP}_{q}(f)$ exists, and it is attained for every point in a Zariski open dense subset of the space of nondegenerate polynomials with fixed Newton polygon $\Delta$. This polygon does not depend on $q$, only on $p$, we denote it $\operatorname{GNP}(\Delta, p)$. In the one dimensional case, one can calculate this polygon explicitely [4, [16], and the Hasse polynomial, determining the hypersurface of the space of polynomials outside which we have $\mathrm{NP}_{q}(f)=\operatorname{GNP}(\Delta, p)$.

Let us speak about the behaviour of the generic Newton polygon when $p$ varies. From ramification theory, one can easily see that a necessary condition for this polygon to coincide with the Hodge polygon is $p \equiv 1 \bmod D$. Adolphson and Sperber conjectured this condition is sufficient. This is true when $n \leq 3$, but in higher dimensions one has to replace $D$ by a (generally strict) multiple $D^{*}$, as shown in [18, 19. We can rewrite this result

$$
\liminf _{p \rightarrow \infty} \operatorname{GNP}(\Delta, p)=\operatorname{HP}(\Delta) .
$$

Wan conjectured [19, Conjecture 1.11] that under certain extra hypotheses the limit exists, that is

$$
\lim _{p \rightarrow \infty} \operatorname{GNP}(\Delta, p)=\operatorname{HP}(\Delta)
$$

This result is known for one variable Laurent polynomials [4, 16]. In this paper we prove this conjecture for the following families of polyhedra

i/ $\Delta$ is the convex hull of points $\left\{d_{i} \mathbf{e}_{i},-d_{i}^{\prime} \mathbf{e}_{i}\right\}_{1 \leq i \leq n}$, where $\left(\mathbf{e}_{1}, \ldots, \mathbf{e}_{n}\right)$ forms a basis for the $\mathbb{Z}$-module $\mathbb{Z}^{n}$, and the $d_{i}, d_{i}^{\prime}$ are nonnegative integers, $\left(d_{i}, d_{i}^{\prime}\right) \neq$ $(0,0)$; this is Theorem 3.1

ii) $\Delta$ is the convex hull of points $\left\{d_{i} \mathbf{f}_{i},-d_{i}^{\prime} \mathbf{f}_{i}\right\}_{1 \leq i \leq n}$, where $\mathbf{f}_{1}, \ldots, \mathbf{f}_{n}$ generate a submodule $M$ of $\mathbb{Z}^{n}$ such that $2 \mathbb{Z}^{n} \subset M$; see Theorem 5.1.

To our knowledge, these are the first results on the asymptotic behaviour of Newton polygons associated to higher dimensional character sums, when the denominator $D$ is neither 1 nor 2 . Note that the first case implies the conjecture for the polynomials studied by Deligne.

Another, more difficult, question, is the following: choose a Laurent polynomial $\widetilde{f}$ whose coefficients are in $\overline{\mathbb{Q}}$; denote by $\mathbb{Q}_{\tilde{f}}$ the extension of $\mathbb{Q}$ generated by the coefficients of $\widetilde{f}$. For each prime $p$ in $\mathbb{Q}$, choose $\mathfrak{p}$ a prime dividing $p$ in the field $\mathbb{Q}_{\tilde{f}}$, with residue field $\mathbb{F}_{q}$. One can ask for the variation of the Newton polygons $\operatorname{NP}_{q}(\tilde{f}$ $\bmod \mathfrak{p}$ ) of the reductions modulo $\mathfrak{p}$ of $\tilde{f}$ when $p$ tends to infinity. Consider the space of polynomials with coefficients in $\overline{\mathbb{Q}}$, with fixed simplicial Newton polyhedron $\Delta$, and having non zero coefficients for some monomials such that the sub monoid of 
$M_{\Delta}$ generated by their exponents contains all the points in $M_{\Delta}$ except a finite number. In this situation Wan conjectures [19, Conjecture 1.12] that there is a (Zariski) dense open subset defined over $\mathbb{Q}$ of the space of these polynomials such that for any $\tilde{f}$ in this open we have

$$
\lim _{p \rightarrow \infty} \operatorname{NP}_{q}(\widetilde{f} \bmod \mathfrak{p})=\operatorname{HP}(\Delta) .
$$

This result is known for the space of polynomials of degree $d$ in one variable 21, Theorem 1.3], and for the space of Laurent polynomials of degrees $d$ and $d^{\prime}$ in one variable [15]. We will show it for certain spaces of Laurent polynomials whose Newton polyhedron has one of the forms described above (cases $i /$, ii $/$ ): see Theorems 3.2 and 5.2 .

When the multiplicative character $\chi$ is no longer trivial, the situation is quite different. Adolphson and Sperber have shown (under the same non degeneracy conditions) that the $L$-function has the same degree as in the purely additive case (cf. [2], 3]). They also gave a lower bound for Newton polygons associated to such sums. Again, one can describe this lower bound in terms of Poincaré series, but in this new situation it depends on the residue of $p$ modulo the order of the character $\chi$. As a consequence, one cannot hope a limit for the generic Newton polygons when $p$ tends to infinity, except when the order of $\chi$ is two (actually this particular case gives the case ii/ above). We get a weaker result in this case: when $p$ goes to $\infty$ along a residue class modulo the order of the multiplicative character, we still have a limit. This is a generalisation of the one dimensional results in [5]. See theorems 4.1 and 4.2 for more details.

This paper is organized as follows: in the first chapter, we use the free sum of convex polytopes, and express the associated Hodge polygon from the Hodge polygons of the factors. In the second, we use results from $\ell$-adic cohomology (mostly Künneth formula) to express Newton polygons associated to ( $L$-functions from character sums associated to) certain polynomials in several variables from Newton polygons associated to one variable polynomials. In the third chapter, we show Wan's conjectures in case $i /$ : we recall briefly the one variable situation, from which we deduce theorems 3.1 and 3.2. In chapter 4 , we give some applications to sums twisted by a multiplicative character, and show the existence of a limit when one restricts to residual classes. Finally, we use the preceding results, and Poisson's formula to show case ii/ of Wan's conjectures in the last chapter.

\section{Notations.}

In order to make the reader more comfortable, and since we shall use several operations on convex polygons, we group here the different notations and for each we give the reference in the text. Let $\Pi_{1}, \Pi_{2}$ be polygons, and $a>0$ a real number

$$
\begin{array}{clc}
\Pi_{1} \times \Pi_{2} & \text { product } & \text { Definition } 1.2 \\
\Pi_{1}+\Pi_{2} & \text { sum } & \text { Definition } 4.1 \\
a \Pi_{1} & \text { vertical scaling } & \text { Definition } 4.1 \\
\Pi_{1} \Pi_{2} & \text { juxtaposition } & \text { Definition } 5.2
\end{array}
$$




\section{Free sums of polytopes And their Hodge polygons.}

In this chapter, we fix once and for all two convex polytopes $\Delta_{1}$ in $\mathbb{R}^{n_{1}}$ and $\Delta_{2}$ in $\mathbb{R}^{n_{2}}$. We recall the definition of their free sum $\Delta_{1} \oplus \Delta_{2}$, and give some of its properties; then we express the Poincaré series of the graded algebra $\mathcal{A}_{\Delta_{1} \oplus \Delta_{2}}$ from those of $\mathcal{A}_{\Delta_{1}}$ and $\mathcal{A}_{\Delta_{2}}$, in order to express the Hodge polygon of $\Delta_{1} \oplus \Delta_{2}$ from those of $\Delta_{1}$ and $\Delta_{2}$.

We first describe the free sum of polytopes (cf. [11, 16.1.3]); in the following we suppose that our polytopes always generate the ambient affine space; moreover the dimension of a polytope is the dimension of the smallest affine space containing it, and we assume the dimension of the empty polytope to be -1 .

Definition 1.1. Let $\Delta_{1}$ and $\Delta_{2}$ be two convex polytopes, lying respectively in $\mathbb{R}^{n_{1}}$ and $\mathbb{R}^{n_{2}}$. Their free sum is the convex polytope which is the convex hull of $\Delta_{1} \times$ $\{0\} \cup\{0\} \times \Delta_{2}$ in $\mathbb{R}^{n_{1}+n_{2}}$. We denote it by $\Delta_{1} \oplus \Delta_{2}$.

Remark 1.1. There is a difference between the operation we have just defined and the usual (or Minkowski) sum of polytopes. For instance, if $\Delta_{1}=\left[0, d_{1}\right] \subset \mathbb{R}$ and $\Delta_{2}=\left[0, d_{2}\right] \subset \mathbb{R}$, then their free sum $\Delta_{1} \oplus \Delta_{2}$ is the triangle with vertices $(0,0),\left(d_{1}, 0\right)$ and $\left(0, d_{2}\right)$, but their usual sum is the rectangle with vertices $(0,0),\left(d_{1}, 0\right),\left(0, d_{2}\right)$ and $\left(d_{1}, d_{2}\right)$.

We now determine the facets not containing the origin of the free sum of two polytopes, each one containing the origin. These results must be well known, but since we did not find a suitable reference, and many proofs in this paper depend on it, we recall them here.

By their very definition, the facets of a polytope are its intersections with its supporting hyperplanes, but also the whole polytope and the empty set $\emptyset$; the last two ones are generally called the "improper facets".

Proposition 1.1. Let $\Delta_{1}$ and $\Delta_{2}$ be two convex polytopes containing the origin, and $\Delta:=\Delta_{1} \oplus \Delta_{2}$ be their free sum. The facets of $\Delta$ not containing the origin are the polytopes $\sigma:=\sigma_{1} \oplus \sigma_{2}, \sigma_{i}$ being a facet of $\Delta_{i}$ not containing the origin for $i=1,2$.

Moreover, if for each $i=1,2$ the facet $\sigma_{i}$ has dimension $d_{i}$, then $\sigma$ is a facet of $\Delta$ of dimension $d_{1}+d_{2}+1$.

Proof. First we show that if $\sigma_{1}$ (resp. $\sigma_{2}$ ) is a facet (perhaps empty) of $\Delta_{1}$ (resp. $\Delta_{2}$ ) not containing the origin, then $\sigma_{1} \oplus \sigma_{2}$ is a facet of $\Delta$ not containing the origin. Let $H_{1}$ with equation $\sum_{i=1}^{n_{1}} a_{i} x_{i}=1$ (resp. $H_{2}$ with equation $\left.\sum_{i=1}^{n_{2}} b_{i} y_{i}=1\right)$ be a supporting hyperplane of $\Delta_{1}\left(\right.$ resp. $\left.\Delta_{2}\right)$ for the facet $\sigma_{1}$ (resp. $\left.\sigma_{2}\right)$. For an empty facet we take all the $a_{i}$ equal to 0 . Since $\Delta_{1}$ contains the origin, it is contained in the half-space $H_{1}^{-}=\left\{\left(x_{1}, \ldots, x_{n_{1}}\right), \sum_{i=1}^{n_{1}} a_{i} x_{i} \leq 1\right\}$ in $\mathbb{R}^{n_{1}}$, and the same is true for $\Delta_{2}$ and $H_{2}^{-}$in $\mathbb{R}^{n_{2}}$. Consider the hyperplane $H$ in $\mathbb{R}^{n_{1}+n_{2}}$ having equation $\sum_{i=1}^{n_{1}} a_{i} x_{i}+\sum_{i=1}^{n_{2}} b_{i} y_{i}=1$. From the definition of $\Delta$ as a convex hull, this polytope is contained in the half-space $H^{-}$of $\mathbb{R}^{n_{1}+n_{2}}$, and the intersection $H \cap \Delta$ must contain $\sigma_{1} \times\{0\}$ and $\{0\} \times \sigma_{2}$. As a consequence, $H$ is a supporting hyperplane of $\Delta$, and the facet $\sigma:=H \cap \Delta$ it determines contains the convex hull of $\sigma_{1} \times\{0\} \cup\{0\} \times \sigma_{2}$, i.e. the free sum $\sigma_{1} \oplus \sigma_{2}$.

To show the reverse inclusion, choose a point $\mathbf{z}\left(z_{1}, \ldots, z_{n_{1}+n_{2}}\right)$ in $\sigma$. Then $\mathbf{z}$ is in $\Delta$, a barycenter of $(\mathbf{x}, 0)$ and $(0, \mathbf{y})$ two points respectively in $\Delta_{1} \times\{0\}$ and $\{0\} \times \Delta_{2}$; 
thus there exists a real number $\lambda \in[0,1]$ such that $z_{i}=\lambda x_{i}$ for $1 \leq i \leq n_{1}$ and $z_{n_{1}+i}=(1-\lambda) y_{i}$ for $1 \leq i \leq n_{2}$. As long as $\mathbf{z}$ is in $H$, we have $\sum a_{i} z_{i}+\sum b_{i} z_{n_{1}+i}=$ 1 ; on the other hand we have $\sum_{i=1}^{n_{1}} a_{i} x_{i} \leq 1$ and $\sum_{i=1}^{n_{2}} b_{i} y_{i} \leq 1$. These last inequalities must be equalities, in other words $\mathbf{x} \in \sigma_{1}, \mathbf{y} \in \sigma_{2}$, and $x \in \sigma_{1} \oplus \sigma_{2}$. We have thus shown that $\sigma=\sigma_{1} \oplus \sigma_{2}$ is a facet of $\Delta$ not containing the origin.

Conversely let $\sigma$ be a facet of $\Delta$ not containing the origin, and $H$ a supporting hyperplane for it. Since $\sigma$ is a convex polytope, it is the convex hull of its extremal points, which are the extremal points of $\Delta$ contained in $\sigma$. Now from their definition, the extremal points of $\Delta$ have the form $\left(\mathbf{x}_{1}, 0\right)$ or $\left(0, \mathbf{x}_{2}\right)$, where $\mathbf{x}_{i}$ describes the extremal points of $\Delta_{i}$. Denote by $S_{1}$ the set of extremal points of $\sigma$ of the first type, and $S_{2}$ the set of extremal points of $\sigma$ of the second type. Then $\sigma$ is the convex hull of $S_{1} \cup S_{2}$, in other words it is the free sum of $\sigma_{1}$ and $\sigma_{2}$, where $\sigma_{i}$ is the convex hull of $S_{i}$, and it is sufficient to show that $\sigma_{i}$ is a facet of $\Delta_{i}$. If $S_{i}=\emptyset$, there is nothing to prove; else from its construction $H_{i}=H \cap \mathbb{R}^{n_{i}}$ is a supporting hyperplane for $\Delta_{i}$ in $\mathbb{R}^{n_{i}}$, and $\sigma_{i}=\Delta \cap H_{i}$ is a facet of $\Delta_{i}$.

We end the proof with the assertion on the dimension: the facet $\sigma_{i}$ is the convex hull of a set $S_{i}$ of points not containing the origin, among which we can choose a maximal subset $S_{i}^{\prime}$ with $d_{i}+1$ affinely independent points. The sets in affine space $\mathbb{R}^{n_{1}+n_{2}}, T_{1}=S_{1}^{\prime} \times\{0\}$ and $T_{2}=\{0\} \times S_{2}^{\prime}$ are disjoint, and affinely independent from their definition. The set $T=T_{1} \cup T_{2}$ is a maximal subset of $d_{1}+d_{2}+2$ affinely independent points in $S=S_{1} \times\{0\} \cup\{0\} \times S_{2}$; finally since $\sigma$ is the convex hull of $S$, we get the last assertion.

Since $\Delta_{i}$ contains the origin, its facets not containing it have dimension at most $n_{i}-1$; from this we deduce the

Corollary 1.1. The codimension 1 facets of $\Delta$ not containing the origin are the $\sigma=\sigma_{1} \oplus \sigma_{2}$, where $\sigma_{i}$ is a codimension 1 facet of $\Delta_{i}$ not containing the origin.

Moreover if $H_{1}$ having equation $\sum a_{i} x_{i}=1$ (resp. $H_{2}$ having equation $\sum b_{i} y_{i}=$ 1 ) is the supporting hyperplane of $\Delta_{1}$ along $\sigma_{1}$ (resp. of $\Delta_{2}$ along $\sigma_{2}$ ), then the supporting hyperplane of $\Delta$ along $\sigma$ has equation $\sum a_{i} x_{i}+\sum b_{i} y_{i}=1$.

We shall now express the different objects associated to the polytope $\Delta$ in the introduction from those associated to each of the $\Delta_{i}$. First we give an alternative definition of the weight: let $\mathbf{u}\left(u_{1}, \ldots, u_{n_{1}+n_{2}}\right)$ be a point in $C(\Delta)$. The half-line $\mathbb{R}^{+} \mathbf{u}$ meets the boundary of the polytope $\Delta$ in a point of a codimension 1 facet $\sigma$ not containing the origin. If $H$ is the supporting hyperplane for this facet, having equation $a_{1} x_{1}+\cdots+a_{n_{1}+n_{2}} x_{n_{1}+n_{2}}=1$, the weight of $\mathbf{u}$ is

$$
w_{\Delta}(\mathbf{u})=a_{1} u_{1}+\cdots+a_{n_{1}+n_{2}} u_{n_{1}+n_{2}} .
$$

Lemma 1.1. Let $\Delta_{1}$ and $\Delta_{2}$ be two convex polytopes containing the origin, and $\Delta:=\Delta_{1} \oplus \Delta_{2}$ be their free sum. Denote by $\sigma_{i}$ a facet of $\Delta_{i}$. Then

$\mathrm{i} /$ the cone $C\left(\sigma_{1} \oplus \sigma_{2}\right)$ in $\mathbb{R}^{n_{1}+n_{2}}$ is the product $C\left(\sigma_{1}\right) \times C\left(\sigma_{2}\right)$;

ii/ the monoid $M_{\Delta}$ is the monoid $M_{\Delta_{1}} \times M_{\Delta_{2}}$ in $\mathbb{Z}^{n_{1}+n_{2}}$;

iii/ the weight $w_{\Delta}$ is the map $w_{\Delta_{1}}+w_{\Delta_{2}}$ from $C(\Delta)$ to $\mathbb{R}_{+}$sending the point $\mathbf{u}=\left(\mathbf{u}_{1}, \mathbf{u}_{2}\right)$ to $w_{\Delta_{1}}\left(\mathbf{u}_{1}\right)+w_{\Delta_{2}}\left(\mathbf{u}_{2}\right)$

iv/ the denominator $D$ of $\Delta$ is the least common multiple of the denominators $D_{1}$ and $D_{2}$ of $\Delta_{1}$ and $\Delta_{2}$. 
Proof. First we show assertion $i$ : the point $\mathbf{u}\left(\mathbf{u}_{1}, \mathbf{u}_{2}\right)$ is in the cone $C\left(\sigma_{1} \oplus \sigma_{2}\right)$ if and only if one can choose a real number $\rho$ such that $\left(\rho \mathbf{u}_{1}, \rho \mathbf{u}_{2}\right)$ is in $\sigma_{1} \oplus \sigma_{2}$. From its definition, this polytope contains exactly the points $\left(\lambda \mathbf{x}_{1},(1-\lambda) \mathbf{x}_{2}\right), \lambda \in[0,1]$, $\mathbf{x}_{i} \in \sigma_{i}$. Each $\mathbf{u}_{i}$ is in $C\left(\sigma_{i}\right)$, and the converse is trivial.

The second assertion is an easy consequence of the definition of the monoid associated to a convex polytope, and assertion $i /$, applied to $\sigma_{i}=\Delta_{i}$.

To show iii/, we use Corollary 1.1 and the description of the weight given above. Let $\sigma$ be a codimension 1 facet of $\Delta$ not containing the origin, such that the half line $\mathbb{R}_{+} \mathbf{u}$ meets the boundary of $\Delta$ in a point of $\sigma$. From Corollary 1.1, we have $\sigma=\sigma_{1} \oplus \sigma_{2}, \sigma_{i}$ being a codimension 1 facet of $\Delta_{i}$ not containing the origin. As a consequence, if $\mathbf{u}=\left(\mathbf{u}_{1}, \mathbf{u}_{2}\right)$, assertion $i /$ ensures that $\mathbf{u}_{i} \in C\left(\sigma_{i}\right)$. If we note $H_{1}: a_{1} x_{1}+\cdots+a_{n_{1}} x_{n_{1}}=1$ (resp. $\left.H_{2}: a_{n_{1}+1} x_{n_{1}+1}+\cdots+a_{n_{1}+n_{2}} x_{n_{1}+n_{2}}=1\right)$ the supporting hyperplane for $\Delta_{1}$ along $\sigma_{1}$ (resp. for $\Delta_{2}$ along $\left.\sigma_{2}\right)$ and if $\mathbf{u}_{1}\left(u_{1}, \ldots, u_{n_{1}}\right)$ $\left(\right.$ resp. $\left.\mathbf{u}_{2}\left(u_{n_{1}+1}, \ldots, u_{n_{1}+n_{2}}\right)\right)$, we must have $w_{\Delta_{1}}\left(\mathbf{u}_{1}\right)=a_{1} u_{1}+\cdots+a_{n_{1}} u_{n_{1}}$ (resp. $\left.w_{\Delta_{2}}\left(\mathbf{u}_{2}\right)=a_{n_{1}+1} u_{n_{1}+1}+\cdots+a_{n_{1}+n_{2}} u_{n_{1}+n_{2}}\right)$. Using Corollary 1.1 again, the equation of the supporting hyperplane for $\Delta$ along $\sigma$ is $a_{1} x_{1}+\cdots+a_{n_{1}+n_{2}} x_{n_{1}+n_{2}}=$ 1 , and $w_{\Delta}(\mathbf{u})=a_{1} u_{1}+\cdots+a_{n_{1}+n_{2}} u_{n_{1}+n_{2}}$; this is the desired result.

The last assertion is a direct consequence of $i i i /$ and the definition of the denominator of a convex polytope.

We deduce from assertion $i$ ii that the algebra $\mathcal{A}_{\Delta}$ is isomorphic to the tensor product (over $k$ ) of the algebras $\mathcal{A}_{\Delta_{1}}$ and $\mathcal{A}_{\Delta_{2}}$. Let us look at the grading; if $\mathbf{x}_{i}^{\mathbf{u}_{i}}$ is in $\mathcal{A}_{\Delta_{i}, \frac{k_{i}}{D_{i}}}$, from assertion iii/, the monomial $\mathbf{x}^{\mathbf{u}}=\mathbf{x}_{1}^{\mathbf{u}_{1}} \mathbf{x}_{2}^{\mathbf{u}_{2}}$ is in $\mathcal{A}_{\Delta, \frac{k}{D}}$, with

$$
\frac{k_{1}}{D_{1}}+\frac{k_{2}}{D_{2}}=\frac{k}{D}
$$

We get the following decomposition for each piece of the grading of $\mathcal{A}_{\Delta}$ :

$$
\mathcal{A}_{\Delta, \frac{k}{D}}=\bigoplus_{\frac{k_{1}}{D_{1}}+\frac{k_{2}}{D_{2}}=\frac{k}{D}} \mathcal{A}_{\Delta_{1}, \frac{k_{1}}{D_{1}}} \otimes \mathcal{A}_{\Delta, \frac{k_{2}}{D_{2}}}
$$

the factorisation of the Poincaré series of $\mathcal{A}_{\Delta}$ with the ones of $\mathcal{A}_{\Delta_{1}}$ and $\mathcal{A}_{\Delta_{2}}$

$$
P_{\mathcal{A}_{\Delta}}(t)=P_{\mathcal{A}_{\Delta_{1}}}\left(t^{\frac{D}{D_{1}}}\right) P_{\mathcal{A}_{\Delta_{2}}}\left(t^{\frac{D}{D_{2}}}\right)
$$

and finally the factorisation $P_{\Delta}(t)=P_{\Delta_{1}}\left(t^{\frac{D}{D_{1}}}\right) P_{\Delta_{2}}\left(t^{\frac{D}{D_{2}}}\right)$.

We end this section showing, from the formula above, that the Hodge polygon of the free sum $\Delta=\Delta_{1} \oplus \Delta_{2}$ can be expressed from the polygons of each factor. In order to show this, we introduce a new operation on convex polytopes. Recall that we have chosen to note a convex polygon of length $a$ starting at $O$ by $\left(s_{i}\right)_{1 \leq i \leq a}$ when it is the juxtaposition of segments of (horizontal) length 1 and slope $s_{i}$.

Definition 1.2. Let $\Pi_{1}$ and $\Pi_{2}$ be two convex polygons. If

$$
\Pi_{1}=\left(s_{i}\right)_{1 \leq i \leq a}, \Pi_{2}=\left(s_{i}^{\prime}\right)_{1 \leq i \leq b},
$$

the product of $\Pi_{1}$ and $\Pi_{2}$ (denoted $\Pi_{1} \times \Pi_{2}$ ) is the convex polygon starting at $O$ defined by

$$
\Pi=\left(s_{i}+s_{j}^{\prime}\right)_{1 \leq i \leq a, 1 \leq j \leq b} .
$$


Remark that the horizontal length of $\Pi$ is the product of the lengths of $\Pi_{1}$ and $\Pi_{2}$, and that the horizontal length of the segment with slope $s$ in $\Pi$ is

$$
\ell=\sum_{s_{i}+s_{j}^{\prime}=s} \ell_{i} \ell_{j}^{\prime}
$$

where $\ell_{i}\left(\right.$ resp. $\left.\ell_{j}^{\prime}\right)$ is the horizontal length of the segment with slope $s_{i}$ (resp. $s_{j}^{\prime}$ ) in $\Pi_{1}\left(\right.$ resp. $\left.\Pi_{2}\right)$.

We deduce the following description for the Hodge polygon $\operatorname{HP}(\Delta)$ from the Hodge polygons of its factors.

Proposition 1.2. Let $\Delta_{1}$ and $\Delta_{2}$ be convex polytopes, $\Delta$ their free sum. Then the Hodge polygon of $\Delta$ is the product of the Hodge polygons of its factors

$$
\operatorname{HP}(\Delta)=\operatorname{HP}\left(\Delta_{1}\right) \times \operatorname{HP}\left(\Delta_{2}\right) .
$$

Proof. Let $\ell_{k_{1}}^{(1)}$ (resp. $\left.\ell_{k_{2}}^{(2)}\right)$ be the horizontal slope of the segment in $\operatorname{HP}\left(\Delta_{1}\right)$ (resp. $H P\left(\Delta_{2}\right)$ ) with slope $\frac{k_{1}}{D_{1}}$ (resp. $\frac{k_{2}}{D_{2}}$ ). The segment with slope $\frac{k}{D}$ in the product $\operatorname{HP}\left(\Delta_{1}\right) \times \operatorname{HP}\left(\Delta_{2}\right)$ has horizontal length $\ell=\sum \ell_{k_{1}}^{(1)} \ell_{k_{2}}^{(2)}$ where the sum runs over $k_{1}$, $k_{2}$ such that $\frac{k_{1}}{D_{1}}+\frac{k_{2}}{D_{2}}=\frac{k}{D}$. We conclude remarking that the definition of $\operatorname{HP}(\Delta)$ from $P_{\Delta}$, joint with the factorisation of $P_{\Delta}$, gives the same result.

\section{Character sums.}

All along this chapter we choose two Laurent polynomials over $k, f_{1}$ and $f_{2}$ respectively in $n_{1}$ et $n_{2}$ variables. We note the corresponding indeterminates $\mathbf{x}_{1}$ and $\mathbf{x}_{2}$, and $f=\left(f_{1}, f_{2}\right)$ the Laurent polynomial in the $n:=n_{1}+n_{2}$ variables $\mathbf{x}=\left(x_{1}, \ldots, x_{n_{1}+n_{2}}\right)$ defined by $f(\mathbf{x})=f_{1}\left(x_{1}, \ldots, x_{n_{1}}\right)+f_{2}\left(x_{n_{1}+1}, \ldots, x_{n_{1}+n_{2}}\right)$. It is an immediate consequence of Definition 1.1 that if $\Delta_{1}$ and $\Delta_{2}$ denote respectively the Newton polytopes of $f_{1}$ and $f_{2}$ in $\mathbb{R}^{n_{1}}$ and $\mathbb{R}^{n_{2}}$, the Newton polytope of $f$ is $\Delta$, the free sum of $\Delta_{1}$ and $\Delta_{2}$.

The aim of this section is to express the $\ell$-adic cohomology spaces associated to the character sums coming from $f$ in terms of the spaces associated to $f_{1}$ and $f_{2}$; our main tool is Künneth formula. From these results we will deduce bounds on the generic Newton polygon associated to $\Delta$ from those associated to $\Delta_{1}$ and $\Delta_{2}$.

We begin by showing that the non degeneracy of $f_{1}$ and $f_{2}$ implies the non degeneracy of $f$.

Lemma 2.1. Let $f_{1}$ and $f_{2}$ be two Laurent polynomials with respective Newton polytopes $\Delta_{1}$ and $\Delta_{2}$. If $f_{1}$ and $f_{2}$ are non degenerate with respect to their Newton polytopes, then $f=\left(f_{1}, f_{2}\right)$ is non degenerate with respect to $\Delta=\Delta_{1} \oplus \Delta_{2}$.

Proof. Recall that if $f$ is a polynomial with Newton polytope at infinity $\Delta$, and $\sigma$ a facet of the polytope $\Delta$, the polynomial $f_{\sigma}$ is the sum of the monomials $f$ whose exponent is in $\sigma$. Then $f$ is non degenerate with respect to $\Delta$ exactly when for any facet $\sigma$ not containing the origin, the polynomials $\frac{\partial f_{\sigma}}{\partial x_{i}}, 1 \leq i \leq n_{1}+n_{2}$ have no common zero in $\left(\bar{k}^{\times}\right)^{n_{1}+n_{2}}$. From Lemma 1.1] every facet of $\Delta$ not containing the origin can be written $\sigma_{1} \oplus \sigma_{2}$, where $\sigma_{1}$ is a facet of $\Delta_{1}$ (resp. $\sigma_{2}$ a facet of $\Delta_{2}$ ). One easily verifies $f_{\sigma}(\mathbf{x})=f_{1, \sigma_{1}}\left(\mathbf{x}_{1}\right)+f_{2, \sigma_{2}}\left(\mathbf{x}_{2}\right)$ and we have

$$
\frac{\partial f_{\sigma}}{\partial x_{i}}=\left\{\begin{array}{lll}
\frac{\partial f_{1, \sigma_{1}}}{\partial x_{i}} & \text { for } & 1 \leq i \leq n_{1} \\
\frac{\partial f_{2, \sigma_{2}}}{\partial x_{i}} & \text { for } & n_{1}+1 \leq i \leq n_{1}+n_{2}
\end{array}\right.
$$


Thus $\mathbf{x}=\left(\mathbf{x}_{1}, \mathbf{x}_{2}\right)$ is a common zero for the polynomials $\frac{\partial f_{\sigma}}{\partial x_{i}}, 1 \leq i \leq n_{1}+n_{2}$ when $\mathbf{x}_{1}$ is a common zero for the $\frac{\partial f_{1, \sigma_{1}}}{\partial x_{i}}, 1 \leq i \leq n_{1}$ and $\mathbf{x}_{2}$ is a common zero for the $\frac{\partial f_{2, \sigma_{2}}}{\partial x_{i}}, n_{1}+1 \leq i \leq n_{1}+n_{2}$. In other words, non degeneracy of $f_{1}$ and $f_{2}$ implies that of $f$.

Let $\psi$ be a non trivial additive character over $k$, and $\mathcal{L}_{\psi}$ be the $\overline{\mathbb{Q}}_{\ell}$-sheaf over $\mathbb{A}_{k}^{1}$ associated to $\psi$ and the Artin-Schreier covering $y^{q}-y=x$. Let $\chi$ be a multiplicative

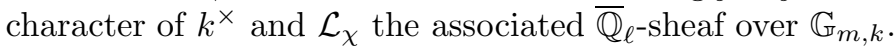

For $X$ a $k$-scheme of finite type, $f$ a regular function over $X$ (i.e. a morphism $f: X \rightarrow \mathbb{A}^{1}$ ), and $g$ a regular function not vanishing over $X$, one can define as in the introduction the function $L(X, f, g ; T)$, and Grothendieck's trace formula allows us to reinterpret it in terms of characteristic polynomials of Frobenius action on the cohomology spaces of the sheaf $f^{*} \mathcal{L}_{\psi} \otimes g^{*} \mathcal{L}_{\chi}$

$$
L(X, f, g ; T)=\prod_{i} \operatorname{det}\left(I-T F \mid H_{c}^{i}\left(X \otimes \bar{k}, f^{*} \mathcal{L}_{\psi} \otimes g^{*} \mathcal{L}_{\chi}\right)\right)^{(-1)^{i-1}} .
$$

We come back to our situation. Consider the three functions $f_{i}: \mathbb{G}_{m}^{n_{i}} \rightarrow \mathbb{A}^{1}$, $1 \leq i \leq 2$, and $f=\left(f_{1}, f_{2}\right): \mathbb{G}_{m}^{n} \rightarrow \mathbb{A}^{1}$. We fix a character $\chi_{1}\left(\right.$ resp. $\left.\chi_{2}\right)$ of $\left(k^{\times}\right)^{n_{1}}$ (resp. $\left.\left(k^{\times}\right)^{n_{2}}\right)$, and we denote by $\chi$ the character $\left(\chi_{1}, \chi_{2}\right)$ of $\left(k^{\times}\right)^{n}$. From the definition of $f$, we have (denoting $\operatorname{pr}_{i}$ the canonical projections of $\mathbb{G}_{m}^{n}=\mathbb{G}_{m}^{n_{1}} \times \mathbb{G}_{m}^{n_{2}}$ on each of its factors) the following: $f^{*} \mathcal{L}_{\psi} \otimes \mathcal{L}_{\chi}=\otimes_{i=1}^{2} \operatorname{pr}_{i}^{*}\left(f_{i}^{*} \mathcal{L}_{\psi} \otimes \mathcal{L}_{\chi_{i}}\right)$ is the tensor product of the sheaves $f_{i}^{*} \mathcal{L}_{\psi} \otimes \mathcal{L}_{\chi_{i}}$. From Künneth formula, we have

$$
H_{c}^{\bullet}\left(\mathbb{G}_{m}^{n}, f^{*} \mathcal{L}_{\psi} \otimes \mathcal{L}_{\chi}\right)=H_{c}^{\bullet}\left(\mathbb{G}_{m}^{n_{1}}, f_{1}^{*} \mathcal{L}_{\psi} \otimes \mathcal{L}_{\chi_{1}}\right) \otimes H_{c}^{\bullet}\left(\mathbb{G}_{m}^{n_{2}}, f_{2}^{*} \mathcal{L}_{\psi} \otimes \mathcal{L}_{\chi_{2}}\right)
$$

Now $f$ is non degenerate exactly when both $f_{1}$ and $f_{2}$ are; in this case we simply rewrite the above relation

$$
H_{c}^{n}\left(\mathbb{G}_{m}^{n}, f^{*} \mathcal{L}_{\psi} \otimes \mathcal{L}_{\chi}\right)=H_{c}^{n_{1}}\left(\mathbb{G}_{m}^{n_{1}}, f_{1}^{*} \mathcal{L}_{\psi} \otimes \mathcal{L}_{\chi_{1}}\right) \otimes H_{c}^{n_{2}}\left(\mathbb{G}_{m}^{n_{2}}, f_{2}^{*} \mathcal{L}_{\psi} \otimes \mathcal{L}_{\chi_{2}}\right)
$$

from [7, Theorem 1.3] when $\chi$ is trivial, [3, Theorem 4.2] in the general case. In other words, the function $L(f, \chi ; T)^{(-1)^{n-1}}$ is the polynomial whose reciprocal roots are the product of couples of reciprocal roots of the polynomials $L\left(f_{1}, \chi_{1} ; T\right)^{(-1)^{n_{1}-1}}$ and $L\left(f_{2}, \chi_{2} ; T\right)^{(-1)^{n_{2}-1}}$.

Recall that for a Laurent polynomial $f$ we denote by $\mathrm{NP}_{q}(f, \chi)$ the Newton polygon of the polynomial $L(f, \chi ; T)^{(-1)^{n-1}}$. We first deduce from the results above a factorisation of $\mathrm{NP}_{q}(f, \chi)$ that we shall use later.

Lemma 2.2. Notations being as above, we have the following equality of Newton polygons

$$
\mathrm{NP}_{q}(f, \chi)=\mathrm{NP}_{q}\left(f_{1}, \chi_{1}\right) \times \mathrm{NP}_{q}\left(f_{2}, \chi_{2}\right) .
$$

For a $n$-dimensional polytope $\Delta$, and $\chi$ a multiplicative character as above, let us define the generic Newton polygon $\operatorname{GNP}(\Delta, \chi, p)$ as the lower bound for the polygons $\mathrm{NP}_{q}(f, \chi)$ when $f$ runs over the non degenerate polynomials with Newton polytope $\Delta$. When $\Delta$ is a free sum, we deduce from Lemma 2.2 a bound for the generic Newton polygon above, from the generic Newton polygons of the factors in the free sum.

Corollary 2.1. Let $\Delta_{1}$ and $\Delta_{2}$ be to convex polytopes, and $\Delta$ their free sum. We have

$$
\operatorname{GNP}\left(\Delta_{1}, \chi_{1}, p\right) \times \operatorname{GNP}\left(\Delta_{2}, \chi_{2}, p\right) \preceq \operatorname{GNP}(\Delta, \chi, p) .
$$


Proof. Grothendieck's specialisation theorem (see for instance [12]) ensures that for any $i$, there is a (Zariski) dense open subset $\mathcal{U}_{\Delta_{i}, \chi_{i}, p}$ of $\mathcal{M}_{\Delta_{i}}$, the coefficient space for the non degenerate polynomials with polytope $\Delta_{i}$, such that for any $f_{i}$ in $\mathcal{U}_{\Delta_{i}, \chi_{i}, p}$, we have $\mathrm{NP}_{q}\left(f_{i}, \chi_{i}\right)=\operatorname{GNP}\left(\Delta_{i}, \chi_{i}, p\right)$. From Lemma 2.1, if both $f_{1}$ and $f_{2}$ are non degenerate, $f=\left(f_{1}, f_{2}\right)$ is also non degenerate with respect to $\Delta$, and we get the inclusion $\mathcal{M}_{\Delta_{1}} \times \mathcal{M}_{\Delta_{2}} \subset \mathcal{M}_{\Delta}$. We deduce from Lemma 2.2 that the lower bound for the polygons $\operatorname{NP}_{q}(f, \chi)$ when $f$ runs over $\mathcal{M}_{\Delta_{1}} \times \mathcal{M}_{\Delta_{2}}$ is the polygon $\operatorname{GNP}\left(\Delta_{1}, \chi_{1}, p\right) \times \operatorname{GNP}\left(\Delta_{2}, \chi_{2}, p\right)$ (and it is attained for the polynomials with coefficients in $\left.\mathcal{U}_{\Delta_{1}, \chi_{1}, p} \times \mathcal{U}_{\Delta_{2}, \chi_{2}, p}\right)$. Finally, from the definition of $\operatorname{GNP}(\Delta, \chi, p)$ as the lower bound for the polygons $\mathrm{NP}_{q}(f, \chi)$ when $f$ describes $\mathcal{M}_{\Delta}$, we get the result.

\section{Asymptotic Behaviour in the additive Case.}

We consider the following situation: let $n$ be a fixed integer, and $\left(\mathbf{e}_{1}, \ldots, \mathbf{e}_{n}\right)$ be a basis for the $\mathbb{Z}$-module $\mathbb{Z}^{n}$. We also choose non negative integers $d_{1}, d_{1}^{\prime}, \ldots, d_{n}, d_{n}^{\prime}$ such that for any $i$ we have $\left(d_{i}, d_{i}^{\prime}\right) \neq(0,0)$ (else the situation we shall describe reduces to a lower dimensional one). We denote by $\Delta$ the convex polytope in $\mathbb{R}^{n}$ which is the convex hull of the points $\left\{d_{i} \mathbf{e}_{i},-d_{i}^{\prime} \mathbf{e}_{i}\right\}_{1 \leq i \leq n}$ and the origin if necessary.

The aim of this section is to show the following result

Theorem 3.1. When $p$ tends to infinity, the generic Newton polygon associated to $\Delta$ and the prime $p, \operatorname{GNP}(\Delta, p)$, tends to the Hodge polygon $\operatorname{HP}(\Delta)$.

We shall give the proof at the end of this section; it is a consequence of the results above and the one dimensional case of this theorem, which is already known. We begin by recalling what is known in this case; the interested reader shall look at [4, [16], 21].

Let $f$ be a Laurent polynomial in the variable $x, f(x)=\sum_{i=-d^{\prime}}^{d} a_{i} x^{i}, a_{-d^{\prime}} a_{d} \neq 0$. Clearly the Newton polytope at infinity associated to $f$ is the segment in $\mathbb{R}$ with endpoints $-d^{\prime}$ and $d$; the weight is given, for any $n \in \mathbb{Z}$, by $w(n)=\max \left(\frac{n}{d},-\frac{n}{d^{\prime}}\right)$. We deduce from its definition that the polygon $\operatorname{HP}\left(\left[-d^{\prime}, d\right]\right)$ has endpoints the origin and the point with coordinates $\left(d+d^{\prime}, \frac{d+d^{\prime}}{2}\right)$, and has a segment of length 1 for each of the following slopes

$$
0,1, \frac{1}{d}, \ldots, \frac{d-1}{d}, \frac{1}{d^{\prime}}, \ldots, \frac{d^{\prime}-1}{d^{\prime}}\left(0, \frac{1}{d}, \ldots, \frac{d-1}{d} \text { if } d^{\prime}=0\right) .
$$

From now on we shall denote $s_{1}, \ldots, s_{d+d^{\prime}}$ these slopes, ordered increasingly. As a consequence we deduce an alternative description of the polygon $\operatorname{HP}\left(\left[-d^{\prime}, d\right]\right)$ : it is the polygon starting at the origin and passing through the points $\left(i, s_{1}+\cdots+s_{i}\right)$ for any $1 \leq i \leq d+d^{\prime}$.

As in [17, one can associate to $f$ a differential operator over a space of overconvergent series, and a Frobenius operator. These operators commute to each other, allowing the following reinterpretation of the function $L(f ; T)$ : it is the characteristic polynomial of the Frobenius operator acting on the first de Rham cohomology space. Now one estimates (for $p$ large enough), the principal parts of the coefficients of its matrix, and give congruences for its minors, i.e. for the coefficients of the $L$-function.

Let $\pi$ be the unique root of the polynomial $X^{p-1}+p$ (in a fixed algebraic closure of the field $\mathbb{Q}_{p}$ of $p$-adic numbers) satisfying the congruence $\psi(1) \equiv 1+\pi\left[\pi^{2}\right]$. We 
have $\mathbb{Q}_{p}(\pi)=\mathbb{Q}_{p}\left(\zeta_{p}\right)$, and we set $K=\mathbb{Q}_{p}\left(\zeta_{p}, \zeta_{q-1}\right)$. For any $a$ in $k$, we write $\widetilde{a}$ for its Teichmüller lifting (if $a=0$, then $\widetilde{a}=0$, else the reduction of $\widetilde{a}$ modulo the maximal ideal is $a$, and $\left.\widetilde{a} \in \boldsymbol{\mu}_{q-1}\right)$. Let $\widetilde{f}$ be the polynomial in $K\left[x, x^{-1}\right]$ obtained from $f$ by lifting its coefficients as above. If we set $L(f ; T)=1+\sum_{i=1}^{d+d^{\prime}} M_{i} T^{i}$, we get, for any $1 \leq i \leq d+d^{\prime}$, the congruence in $K$

$$
M_{i} \equiv u_{i} \mathcal{P}_{d, d^{\prime}, i}^{\rho, \rho^{\prime}}\left(\widetilde{a}_{-d^{\prime}}, \ldots, \widetilde{a}_{d}\right) \pi^{a Y_{i}} \bmod \pi^{a Y_{i}+1},
$$

where $u_{i}$ is an unit in the valuation ring of $K$, and the polynomials $\mathcal{P}_{d, d^{\prime}, i}^{\rho, \rho^{\prime}}$ can be chosen with their coefficients in $\mathbb{Q}$, depending only on the degrees $d$ and $d^{\prime}$, and the respective residues $\rho, \rho^{\prime}$ of $p$ modulo $d, d^{\prime}$.

If $0 \leq i_{1} \leq d, 0 \leq i_{2}<d^{\prime}$ are two integers determined by the condition

$$
\left\{s_{1}, \ldots, s_{i}\right\}=\{0\} \cup\left\{\frac{j}{d}, 1 \leq j \leq i_{1}\right\} \cup\left\{\frac{j}{d^{\prime}}, 1 \leq j \leq i_{2}\right\},
$$

then we can express $Y_{i}$ from the symmetric group on $i$ elements, acting on the set $\left\{-i_{2}, \ldots, 0, \ldots, i_{1}\right\}$ :

$$
Y_{i}=\min _{\sigma \in S_{i}} \sum_{j=-i_{2}}^{i_{1}}\lceil w(p j-\sigma(j))\rceil,
$$

where $\lceil x\rceil$ denotes the least integer greater than or equal to $x$.

As a consequence we get a rather precise description of the one dimensional case for a fixed, sufficiently large prime $p$ : on one hand the generic Newton polygon (with respect to the valuation $\left.v_{q}\right), \operatorname{GNP}\left(\left[-d^{\prime}, d\right], p\right)$, has vertices the origin and the points with coordinates $\left(i, \frac{Y_{i}}{p-1}\right)_{1 \leq i \leq d+d^{\prime}}$, on the other hand the reduction modulo $p$ of the Hasse polynomial

$$
\mathcal{H}_{\left[-d^{\prime}, d\right]}^{\rho, \rho^{\prime}}\left(\widetilde{a}_{-d^{\prime}}, \ldots, \widetilde{a}_{d}\right):=\prod_{i=1}^{d+d^{\prime}} \mathcal{P}_{d, d^{\prime}, i}^{\rho, \rho^{\prime}}\left(\widetilde{a}_{-d^{\prime}}, \ldots, \widetilde{a}_{d}\right),
$$

defines a hypersurface in the space of Laurent polynomials of degrees $d, d^{\prime}$ having their coefficients in $\overline{\mathbb{F}}_{p}$. Every polynomial, defined over $\mathbb{F}_{q}$, whose coefficients do not belong to this hypersurface satisfies $\mathrm{NP}_{q}(f)=\mathrm{GNP}\left(\left[-d^{\prime}, d\right], p\right)$.

When $p$ varies, one easily checks that for all $1 \leq i \leq d+d^{\prime}$ we have

$$
\lim _{p \rightarrow \infty} \frac{Y_{i}}{p-1}=s_{1}+\cdots+s_{i}
$$

i.e. the generic Newton polygon converges to the Hodge polygon. Finally, let us define the polynomial (with coefficients in $\mathbb{Q}$ )

$$
\mathcal{H}_{\left[-d^{\prime}, d\right]}\left(X_{-d^{\prime}}, \ldots, X_{d}\right)=\prod_{\left(\rho, \rho^{\prime}\right) \in(\mathbb{Z} / d \mathbb{Z})^{\times} \times\left(\mathbb{Z} / d^{\prime} \mathbb{Z}\right)^{\times}} \mathcal{H}_{\left[-d^{\prime}, d\right]}^{\rho, \rho^{\prime}}\left(X_{-d^{\prime}}, \ldots, X_{d}\right) .
$$

If $f \in \overline{\mathbb{Q}}\left[x, x^{-1}\right]$ is a Laurent polynomial with $\Delta(f)=\left[-d^{\prime}, d\right]: f(x)=\sum_{i=-d^{\prime}}^{d} A_{i} x^{i}$, and if we choose for any prime $p$ a prime $\mathfrak{p}$ above $p$ in the ring of integers of the extension $\mathbb{Q}_{f}$ of $\mathbb{Q}$ defined by the coefficients of $f$, we have $\lim _{p \rightarrow \infty} \mathrm{NP}_{q}(f$ $\bmod \mathfrak{p})=\operatorname{HP}\left(\left[-d^{\prime}, d\right]\right)$ as long as

$$
\mathcal{H}_{\left[-d^{\prime}, d\right]}\left(A_{-d^{\prime}}, \ldots, A_{d}\right) \neq 0
$$

In other words there exists a Zariski dense open subset $\mathcal{U}$, defined over $\mathbb{Q}$, of the space of Laurent polynomials in one variable over $\overline{\mathbb{Q}}$ having Newton polyhedron at 
infinity $\left[-d^{\prime}, d\right]$, such that for any $f$ in $U$, the $\operatorname{limit}_{p \rightarrow \infty} \lim _{q}(f \bmod \mathfrak{p})$ exists and is exactly the Hodge polygon.

We are ready show Theorem 3.1 .

Proof. We begin by reducing the problem to the following case: $\mathbf{e}_{i}$ is the $i$-th vector of the canonical basis of $\mathbb{R}^{n}$. One can define a left action of $\mathbf{M}_{n}(\mathbb{Z})$ on the space of Laurent polynomials in $n$ variables, associating to the polynomial $f(\mathbf{x})=\sum a_{\mathbf{i}} \mathbf{x}^{\mathbf{i}}$ and the matrix $M$ the polynomial ${ }^{M} f(\mathbf{x})=f\left({ }^{M} \mathbf{x}\right)=\sum a_{\mathbf{i}}\left({ }^{M} \mathbf{x}\right){ }^{\mathbf{i}}=\sum a_{\mathbf{i}} \mathbf{x}^{M \mathbf{i}}$, where ${ }^{M} \mathbf{X}$ is the $n$-tuple of variables with $i$-th equal to $\prod_{j=1}^{n} x_{j}^{m_{j i}}$, and $M \mathbf{i}$ is the usual multiplication of $M$ and the column vector i. If $M \in \mathbf{G L}_{n}(\mathbb{Z})$, the map $\mathbf{x} \mapsto^{M} \mathbf{x}$ is one-to-one over $\left(k^{\times}\right)^{n}$, and all its extensions. Thus we get $L\left({ }^{M} f ; T\right)=L(f ; T)$.

Let now $f$ be a Laurent polynomial of the form $f(\mathbf{x})=\sum_{i=1}^{n} \sum_{j=-d_{i}^{\prime}}^{d_{i}} a_{i j} \mathbf{x}^{j \mathbf{e}_{i}}$, whose Newton polyhedron at infinity is $\Delta$. Choosing for $M$ the base change matrix from $\left\{\mathbf{e}_{1}, \ldots, \mathbf{e}_{n}\right\}$ to the canonical basis, we get ${ }^{M} f(\mathbf{x})=\sum_{i=1}^{n} \sum_{j=-d_{i}^{\prime}}^{d_{i}} a_{i j} x_{i}^{j}$, whose associated Newton polyhedron is the convex hull of the points with coordinates

$$
\left(d_{1}, 0, \ldots, 0\right),\left(-d_{1}^{\prime}, 0, \ldots, 0\right), \ldots,\left(0, \ldots, 0, d_{n}\right),\left(0, \ldots, 0,-d_{n}^{\prime}\right),
$$

and the origin. This polytope is the free sum of the segments $\left[-d_{i}^{\prime}, d_{i}\right], 1 \leq i \leq n$, and Corollary 2.1 ensures us

$$
\operatorname{GNP}\left(\left[-d_{1}^{\prime}, d_{1}\right], p\right) \times \cdots \times \operatorname{GNP}\left(\left[-d_{n}^{\prime}, d_{n}\right], p\right) \preceq \operatorname{GNP}(\Delta, p) .
$$

On the other hand, from Proposition [1.2, we have $\operatorname{HP}(\Delta)=\operatorname{HP}\left(\left[-d_{1}^{\prime}, d_{1}\right]\right) \times$ $\cdots \times \operatorname{HP}\left(\left[d_{n}^{\prime}, d_{n}\right]\right)$. The result comes from the fact that for each $i$, the polygon $\operatorname{GNP}\left(\left[-d_{i}^{\prime}, d_{i}\right], p\right)$ tends to $\operatorname{HP}\left(\left[-d_{i}^{\prime}, d_{i}\right]\right)$ when $p$ tends to $\infty$ : the polygon $\operatorname{GNP}(\Delta, p)$ is between two polygons having the same limit.

Remark 3.1. In the case $p \equiv 1$ modulo $\operatorname{lcm}\left(d, d^{\prime}\right)$, we know (cf. 17) that the polygons $\operatorname{GNP}\left(\left[-d^{\prime}, d\right], p\right)$ and $\operatorname{HP}\left(\left[-d^{\prime}, d\right]\right)$ coincide. As a consequence, when $p \equiv 1$ modulo $D=\operatorname{lcm}\left(d_{i}, d_{i}^{\prime}\right)_{1 \leq i \leq n}$, the polygons $\operatorname{GNP}(\Delta, p)$ and $\operatorname{HP}(\Delta)$ coincide, and the Adolphson-Sperber conjecture [1, p. 386] is true in this case.

Let us consider the second question, that is: does there exist a dense open subset $\mathcal{U}_{\Delta}$, defined over $\mathbb{Q}$, in the space of polynomials having their coefficients in $\overline{\mathbb{Q}}$, and Newton polyhedron $\Delta$ such that for any $f$ in $\mathcal{U}_{\Delta}$, we have $\lim _{p \rightarrow \infty} \mathrm{NP}_{q}(f \bmod \mathfrak{p})=$ $\operatorname{HP}(\Delta)$, where $\mathfrak{p}$ is a prime above $p$ in the ring of integers of the extension $\mathbb{Q}_{f}$ generated by the coefficients of $f$. Since we do not consider all polynomials having Newton polygon at infinity $\Delta$, we cannot answer this question. But concerning the subfamilies we have considered, we get the following result

Theorem 3.2. There exists a dense open subset $\mathcal{U}$ defined over $\mathbb{Q}$ in the space of polynomials of the form $f(x)=\sum_{i=1}^{n} \sum_{j=-d_{i}^{\prime}}^{d_{i}} A_{i j} \mathbf{x}^{j e_{i}}$ with their coefficients in $\overline{\mathbb{Q}}$ such that for any polynomial in $\mathcal{U}$, we have

$$
\lim _{p \rightarrow \infty} \operatorname{NP}_{q}(f \quad \bmod \mathfrak{p})=\operatorname{HP}(\Delta) .
$$

Proof. For a polynomial of the form $f(x)=\sum_{i=1}^{n} \sum_{j=-d_{i}^{\prime}}^{d_{i}} A_{i j} \mathbf{x}^{j \mathbf{e}_{i}}$, we have $\mathrm{NP}_{q}(f$ $\bmod \mathfrak{p})=\operatorname{GNP}\left(\left[-d_{1}^{\prime}, d_{1}^{\prime}\right], p\right) \times \cdots \times \operatorname{GNP}\left(\left[-d_{n}^{\prime}, d_{n}\right], p\right)$ if and only if its coefficients 
satisfy

$$
\prod_{k=1}^{n} \mathcal{H}_{\left[-d_{k}^{\prime}, d_{k}\right]}^{\rho_{k}, \rho_{k}^{\prime}}\left(A_{k,-d_{k}^{\prime}}, \ldots, A_{k, d_{k}}\right) \neq 0 \bmod p,
$$

where $\rho_{k}\left(\right.$ resp. $\left.\rho_{k}^{\prime}\right)$ is the remainder of the euclidean division of $p$ by $d_{k}$ (resp. by $\left.d_{k}^{\prime}\right)$. Note $\mathcal{H}^{\rho}$ this polynomial, with $\rho=\left(\rho_{1}, \rho_{1}^{\prime}, \ldots, \rho_{n}, \rho_{n}^{\prime}\right) \in\left(\mathbb{Z} / d_{1} \mathbb{Z}\right)^{\times} \times \cdots \times$ $\left(\mathbb{Z} / d_{n}^{\prime} \mathbb{Z}\right)^{\times}$. We know that the polynomials $\mathcal{H}_{\left[-d_{k}^{\prime}, d_{k}\right]}^{\rho_{k}, \rho_{k}^{\prime}}$ have their coefficients in $\mathbb{Q}$, hence $\mathcal{H}^{\rho}$ has its coefficients in $\mathbb{Q}$. If we set

$$
\mathcal{H}\left(X_{i j}\right)=\prod_{\rho} \mathcal{H}^{\rho}\left(X_{i j}\right)=\prod_{i=1}^{n} \mathcal{H}_{\left[-d_{i}^{\prime}, d_{i}\right]}\left(X_{i j}\right),
$$

then for any $f$ whose coefficients lie outside the hypersurface with equation $\mathcal{H}=0$, and for any sufficiently large $p$, we have

$$
\mathrm{NP}_{q}(f \bmod \mathfrak{p})=\operatorname{GNP}\left(\left[-d_{1}^{\prime}, d_{1}^{\prime}\right], p\right) \times \cdots \times \operatorname{GNP}\left(\left[-d_{n}^{\prime}, d_{n}\right], p\right),
$$

and we get the result since the last polygon tends to $\operatorname{HP}(\Delta)$.

\section{Asymptotic Behaviour in the tWisted CASE.}

In this section, $\left(\mathbf{e}_{1}, \ldots, \mathbf{e}_{n}\right)$ denotes again a basis for the $\mathbb{Z}$-module $\mathbb{Z}^{n}$, and $\Delta$ the convex polytope in $\mathbb{R}^{n}$ which is the convex hull of the points $\left\{d_{i} \mathbf{e}_{i},-d_{i}^{\prime} \mathbf{e}_{i}\right\}_{1 \leq i \leq n}$ and the origin if necessary. We shall study the asymptotic behaviour of Newton polygons of the form $\mathrm{NP}_{q}(f, \chi), f$ a polynomial with Newton polytope $\Delta$, and $\chi$ a multiplicative character of $k^{\times}$with fixed order. This study has been done in the one dimensional case in [5], and we generalize it here. The results are rather different from the preceding section since we shall not get a unique limit, but a limit for each invertible class modulo the order of the character $\chi$. Note that all results before Theorem 4.1 are true for any polytope $\Delta$.

Denote by $\omega$ the Teichmüller character of $k^{\times}$, which is a generator for the group of characters of $k^{\times}$. For a fixed $n$-tuple $\boldsymbol{\delta}=\left(\delta_{1}, \ldots, \delta_{n}\right)$ of integers, denote by $\chi=\omega^{\boldsymbol{\delta}}$ the character on $\left(k^{\times}\right)^{n}$ defined by $\chi\left(x_{1}, \ldots, x_{n}\right)=\omega\left(x_{1}\right)^{\delta_{1}} \ldots \omega\left(x_{n}\right)^{\delta_{n}}$. Let $f$ be a polynomial with Newton polytope $\Delta$ (generating $\mathbb{R}^{n}$ ), non degenerate. Adolphson and Sperber have shown that the function $L(f, \chi ; T)^{(-1)^{n-1}}$ is a polynomial, having degree $n ! V(\Delta)$; they also gave a lower bound for its Newton polygon [3, Theorem 3.17 , that we shall call in the sequel Hodge polygon associated to $\Delta$ and $\boldsymbol{\delta}$, and denote by $\operatorname{HP}\left(\Delta, \frac{\boldsymbol{\delta}}{q-1}\right)$.

We first describe this polygon, when the polytope $\Delta$ generates $\mathbb{R}^{n}$. If $i$ and $0 \leq \delta \leq q-2$ are two integers, let $\delta^{(i)}$ be the remainder modulo $q-1$ of the integer $p^{i} \delta$; remark that the sequence $\left(\delta^{(i)}\right)_{i}$ is periodic with period a divisor of $a=\log _{p} q$. We also note $\boldsymbol{\delta}^{(i)}=\left(\delta_{1}^{(i)}, \ldots, \delta_{n}^{(i)}\right)$.

Let $N^{(i)}$ be the lattice $\frac{\delta^{(i)}}{q-1}+\mathbb{Z}^{n}$ in $\mathbb{R}^{n}$. We set $M_{\Delta, \boldsymbol{\delta}^{(i)}}:=C(\Delta) \cap N^{(i)}$, and $\mathcal{A}_{\Delta, \boldsymbol{\delta}^{(i)}}$ the $\mathcal{A}_{\Delta}$-module $k\left[x^{M_{\Delta, \delta^{(i)}}}\right]$. There exists a minimal positive integer $D$ such that each of the images of the $M_{\Delta, \boldsymbol{\delta}^{(i)}}$ by $w_{\Delta}$ is contained in $\frac{1}{D} \mathbb{N}$; we call this integer the denominator of $\left(\Delta, \boldsymbol{\delta}^{(i)}\right)$ in the following. Endowed with the weight, $\mathcal{A}_{\Delta, \boldsymbol{\delta}^{(i)}}$ turns to a graded $\mathcal{A}_{\Delta}$-module, to which we associate a Poincaré series and a polynomial $P_{\Delta, \boldsymbol{\delta}^{(i)}}$ as in the introduction. Let $\Pi^{(i)}$ be the polygon coming from this polynomial. Each of the polynomials $P_{\Delta, \boldsymbol{\delta}^{(i)}}$ has degree at most $n D$, and 
satisfies $P_{\Delta, \boldsymbol{\delta}^{(i)}}(1)=n ! V(\Delta)$. In this way we get a family of polygons $\Pi^{(i)}$ indexed by $0 \leq i \leq a$, each one having length $n ! V(\Delta)$.

Definition 4.1. Let $\Pi$ and $\Pi^{\prime}$ denote two polygons having the same length; we denote by $\Pi+\Pi^{\prime}$ the polygon whose slope on the segment $[i, i+1]$ is the sum of the slopes of the polygons $\Pi$ and $\Pi^{\prime}$ on this segment. For a real number $r>0$, we denote by $r \Pi$ the polygon obtained from $\Pi$ by multiplying all its slopes by $r$.

With these notations, we can describe the Hodge polygon as

$$
\operatorname{HP}\left(\Delta, \frac{\delta}{q-1}\right)=\frac{1}{a} \sum_{i=0}^{a-1} \Pi^{(i)} .
$$

We are also able to express the Hodge polygon associated to a free sum of polytopes and two multiplicative characters from the Hodge polygons associated to each factor. It is the transposition of Proposition 1.2 is this new frame, and we omit the proof, which is very similar to the one for the Hodge polygon of the algebra $\mathcal{A}_{\Delta}$.

Proposition 4.1. Let $\Delta_{1}, \Delta_{2}$ be two convex polytopes, respectively in $\mathbb{R}^{n_{1}}$ and $\mathbb{R}^{n_{2}}$, and $\Delta$ be their free sum. If we set

$$
\boldsymbol{\delta}_{1}=\left(\delta_{1}, \ldots, \delta_{n_{1}}\right), \boldsymbol{\delta}_{2}=\left(\delta_{n_{1}+1}, \ldots, \delta_{n_{1}+n_{2}}\right) \text {, and } \boldsymbol{\delta}=\left(\boldsymbol{\delta}_{1}, \boldsymbol{\delta}_{2}\right)=\left(\delta_{1}, \ldots, \delta_{n_{1}+n_{2}}\right) \text {, }
$$

the Hodge polygon $\operatorname{HP}\left(\Delta, \frac{\delta}{q-1}\right)$ is the product of the Hodge polygons of its factors

$$
\operatorname{HP}\left(\Delta, \frac{\boldsymbol{\delta}}{q-1}\right)=\operatorname{HP}\left(\Delta_{1}, \frac{\boldsymbol{\delta}_{1}}{q-1}\right) \times \operatorname{HP}\left(\Delta_{2}, \frac{\boldsymbol{\delta}_{2}}{q-1}\right) .
$$

The one dimensional case has been studied in [5]; the reader wanting more precisions should refer to this paper. In this case, the Hodge polygon can be described from the classical Hodge polygon for additive character sums, and the valuation of the Gauss sum associated to $\chi$, as given by Stickelberger's classical theorem; for this reason we shall call this new polygon the Hodge Stickelberger polygon, and slightly change our notations.

To justify these changes, we begin by recalling the point of view in 5 . The authors study the asymptotic behaviour in the non generic case of Laurent polynomials in one variable of the form $f\left(x^{s}\right)$. Poisson formula's reduces this problem to the following situation: the Laurent polynomial $f \in k\left[x, x^{-1}\right]$ has Newton polytope the segment $\left[-d^{\prime}, d\right]$, and $\chi$ is a multiplicative character of order $s$. It is not necessarily defined over $k$, but over one of its extensions $k^{\prime}$ of cardinality $q^{\prime} \equiv 1$ modulo $s$. If $\omega^{\prime}$ denotes the Teichmüller character for $k^{\prime \times}$, one can write $\chi=\omega^{\prime \delta}$, with $\delta=\frac{\left(q^{\prime}-1\right) r}{s}$ for some integer $1 \leq r \leq s-1$. Now the Hodge polygon $\operatorname{HP}\left(\left[-d^{\prime}, d\right], \frac{\delta}{q^{\prime}-1}\right)$ is the polygon starting at the origin, ending at the point having coordinates $\left(d+d^{\prime}, \frac{d+d^{\prime}}{2}\right)$, and with a segment of length one for each of the following slopes

$$
\frac{1-\lambda}{d}, \ldots, \frac{d-\lambda}{d}, \frac{\lambda}{d^{\prime}}, \ldots, \frac{d^{\prime}-1+\lambda}{d^{\prime}}\left(\frac{1-\lambda}{d}, \ldots, \frac{d-\lambda}{d} \text { if } d^{\prime}=0\right),
$$

where we set $\lambda=\frac{1}{\log _{p}\left(q^{\prime}\right)(p-1)} s_{p}\left(\frac{\left(q^{\prime}-1\right) r}{s}\right)$ with $s_{p}$ denoting the sum of the $p$-digits of the integer $\frac{\left(q^{\prime}-1\right) r}{s}$. Note that it is exactly the valuation (with respect to $v_{q^{\prime}}$ ) of the Gauss sum associated to $\chi$, as given by Stickelberger theorem. 
An other way to express $\lambda$ is the following: if $\sigma_{p}$ is the permutation of the set $\{0, \ldots, s-1\}$ induced by multiplication of $p$ in $\mathbb{Z} / s \mathbb{Z}$, and if $\sigma$ is the cycle with length $\ell$ in $\sigma_{p}$ containing $r$ in its support, then we have $\lambda=\frac{\sum_{j \in \sigma} j}{s \ell}$. A consequence is that $\lambda$ does not depend on $p$ nor $q^{\prime}$, only on its remainder modulo $s$.

Definition 4.2. The polygon we have just described is the Hodge Stickelberger polygon associated to the polytope $\left[-d^{\prime}, d\right]$ and the rational number $\frac{r}{s}$. We denote it by $\mathrm{HS}\left(\left[-d^{\prime}, d\right], \frac{r}{s}, \nu\right)$, where $\nu$ is the remainder of $p$ modulo $s$.

Once again, there exists a generic Newton polygon $\operatorname{GNP}\left(\left[-d^{\prime}, d\right], \chi, p\right)$, and a Hasse polynomial with its coefficients in $\mathbb{Q}$; neither of them depend on the power of $p$ we choose. Moreover, the Hasse polynomial just depends on the respective remainders $\nu, \rho$ and $\rho^{\prime}$ of $p$ modulo $s, d$ and $d^{\prime}$; we denote it by $\mathcal{H}_{\left[-d^{\prime}, d\right], \frac{r}{s}, \nu}^{\rho}$.

The major difference with the additive case is that, for a fixed polytope, there exist several Hodge-Stickelberger polygons, depending on the remainder of $p$ modulo the order of the multiplicative character $\chi$.

As a consequence, there is no hope that the generic Newton polygons converge when $p$ tends to $+\infty$. Nevertheless, when $p$ tends to $+\infty$ along a class in $(\mathbb{Z} / s \mathbb{Z})^{\times}$, we get a limit :

$$
\lim _{\substack{p \rightarrow+\infty \\ p \equiv \nu[s]}} \operatorname{GNP}\left(\left[-d^{\prime}, d\right], \chi, p\right)=\mathrm{HS}\left(\left[-d^{\prime}, d\right], \frac{r}{s}, \nu\right)
$$

On the other hand, if we define $\mathcal{H}_{\left[-d^{\prime}, d\right], \frac{r}{s}, \nu}=\prod_{\rho, \rho^{\prime}} \mathcal{H}_{\left[-d^{\prime}, d\right], \frac{r}{s}, \nu}^{\rho,}$, then for any polynomial $f \in \overline{\mathbb{Q}}\left[x, x^{-1}\right]$ of the form $f(x)=\sum_{i=-d^{\prime}}^{d} A_{i} x^{i}$, and such that

$$
\mathcal{H}_{\left[-d^{\prime}, d\right], \frac{r}{s}, \nu}\left(A_{-d^{\prime}}, \ldots, A_{d}\right) \neq 0
$$

we have $\lim _{\substack{p \rightarrow+\infty \\ p \equiv \nu[s]}} \operatorname{NP}_{q}(f \bmod \mathfrak{p}, \chi)=\mathrm{HS}\left(\left[-d^{\prime}, d\right], \frac{r}{s}, \nu\right)$ (where as above $\mathfrak{p}$ denotes a prime above $p$ in the ring of integers of the field $\mathbb{Q}_{f}$ generated by the coefficients of $f$ ). In other words, there exists a Zariski dense open subset $\mathcal{U}_{\frac{r}{s}, \nu}$, defined over $\mathbb{Q}$ in the space of polynomials with Newton polytope $\left[-d^{\prime}, d\right]$ and coefficients in $\overline{\mathbb{Q}}$, such that for any $f$ in $U_{\frac{r}{s}, \nu}$, the $\operatorname{limit}_{\substack{p \rightarrow+\infty \\ p \equiv \nu[s]}} \operatorname{NP}_{q}(f \bmod \mathfrak{p}, \chi)$ exists and is the Hodge-Stickelberger polygon.

We are ready to pass to higher dimensions. We first define Hodge-Stickelberger polygons in this new frame

Definition 4.3. Let $\Delta$ be a convex polytope, $\frac{\mathbf{r}}{\mathbf{s}}=\left(\frac{r_{1}}{s_{1}}, \ldots, \frac{r_{n}}{s_{n}}\right)$ an n-tuple of rational numbers, $p$ a prime with remainder $\nu$ modulo $s=\operatorname{lcm}\left(s_{1}, \ldots, s_{n}\right)$ and $q$ a power of $p$ such that $q \equiv 1 \bmod s$. The Hodge-Stickelberger polygon associated to these data is

$$
\operatorname{HS}\left(\Delta, \frac{\mathbf{r}}{\mathbf{s}}, \nu\right):=\operatorname{HP}\left(\Delta, \frac{\boldsymbol{\delta}}{q-1}\right)
$$

where $\boldsymbol{\delta}=\left(\frac{(q-1) r_{1}}{s_{1}}, \ldots, \frac{(q-1) r_{n}}{s_{n}}\right)$.

Remark 4.1. One easily checks from the definition that this polygon does not depend on $q$, the power of $p$ we have to choose. Moreover it only depends on the remainder of $p$ modulo $s$, whence our notation.

Proposition 4.1 can be rewritten for Hodge-Stickleberger polygons. If 
i) $\Delta_{1}$ and $\Delta_{2}$ are two convex polytopes, and $\Delta=\Delta_{1} \oplus \Delta_{2}$ is their free sum ;

ii) $\frac{\mathbf{r}_{1}}{\mathbf{s}_{1}}, \frac{\mathbf{r}_{2}}{\mathbf{S}_{2}}$ and $\frac{\mathbf{r}}{\mathbf{s}}=\left(\frac{\mathbf{r}_{1}}{\mathbf{s}_{1}}, \frac{\mathbf{r}_{2}}{\mathbf{S}_{2}}\right)$ are respectively $n_{1}, n_{2}$ and $n$-tuples of rational numbers;

iii/ $\nu$ is an invertible residue modulo $s:=\operatorname{lcm}\left(s_{1}, s_{2}\right)$, and $\nu_{i}$ its image modulo $s_{i}$

then we have

$$
\operatorname{HS}\left(\Delta, \frac{\mathbf{r}}{\mathbf{s}}, \nu\right)=\operatorname{HS}\left(\Delta_{1}, \frac{\mathbf{r}_{1}}{\mathbf{s}_{1}}, \nu_{1}\right) \times \operatorname{HS}\left(\Delta_{2}, \frac{\mathbf{r}_{2}}{\mathbf{s}_{2}}, \nu_{2}\right) .
$$

Recall that $\left(\mathbf{e}_{1}, \ldots, \mathbf{e}_{n}\right)$ is a basis for the $\mathbb{Z}$-module $\mathbb{Z}^{n}$, and $\Delta$ is the convex polytope in $\mathbb{R}^{n}$ defined as the convex hull of the points $\left\{d_{i} \mathbf{e}_{i},-d_{i}^{\prime} \mathbf{e}_{i}\right\}_{1 \leq i \leq n}$ and the origin if necessary. Choose, for any sufficiently large prime $p$, a multiplicative character $\chi_{i}=\omega_{q-1}^{\frac{(q-1) r_{i}}{s_{i}}}$ for $1 \leq i \leq n$, where $q$ is a suitable power $p$. We denote by $\chi=\left(\chi_{1}, \ldots, \chi_{n}\right)$ the character of $\left(\mathbb{F}_{q}^{\times}\right)^{n}$ induced by the $\chi_{i}$, and $\frac{\mathbf{r}}{\mathbf{s}}=\left(\frac{r_{1}}{s_{1}}, \ldots, \frac{r_{n}}{s_{n}}\right)$. Then the polygon

$$
\operatorname{HS}\left(\Delta, \frac{\mathbf{r}}{\mathbf{s}}, \nu\right):=\operatorname{HS}\left(\left[-d_{1}^{\prime}, d_{1}\right], \frac{r_{1}}{s_{1}}, \nu_{1}\right) \times \cdots \times \operatorname{HS}\left(\left[-d_{n}^{\prime}, d_{n}\right], \frac{r_{n}}{s_{n}}, \nu_{n}\right)
$$

only depends on the residue of $p$ modulo $s:=\operatorname{lcm}\left(s_{1}, \ldots, s_{n}\right)$.

With these notations, we transpose Theorems 3.1 and 3.2 to this new frame

Theorem 4.1. When $p$ tends to infinity along the class $\nu$ in $(\mathbb{Z} / s \mathbb{Z})^{\times}$, the generic Newton polygon associated to $\Delta$, the prime $p$ and the character $\chi, \operatorname{GNP}(\Delta, \chi, p)$, tends to the Hodge-Stickelberger polygon $\operatorname{HS}\left(\Delta, \frac{\mathbf{r}}{\mathbf{s}}, \nu\right)$.

Theorem 4.2. There exists a dense open subset $\mathcal{U}$ in the space of polynomials of the form $f(x)=\sum_{i=1}^{n} \sum_{j=-d_{i}^{\prime}}^{d_{i}} a_{i j} \mathbf{x}^{j e_{i}}$ with their coefficients in $\overline{\mathbb{Q}}$ such that for any polynomial in $\mathcal{U}$, we have

$$
\lim _{\substack{p \rightarrow+\infty \\ p \equiv \nu[s]}} \operatorname{NP}_{q}(f \quad \bmod \mathfrak{p}, \chi)=\operatorname{HS}\left(\Delta, \frac{r}{s}, \nu\right) .
$$

Moreover this open is defined over $\mathbb{Q}$.

Remark 4.2. In case $\operatorname{lcm}\left(d, d^{\prime}\right)$ divides all $p$-digits of $\frac{(q-1) r}{s}$ (for instance when $p \equiv 1$ modulo $\operatorname{lcm}\left(d, d^{\prime}, s\right)$ ) one knows (cf. [5. Proposition 3.7]) that the polygons $\operatorname{GNP}\left(\left[-d^{\prime}, d\right], \chi, p\right)$ and $\operatorname{HS}\left(\left[-d^{\prime}, d\right], \frac{r}{s}, 1\right)$ are the same. As a consequence, if for any $1 \leq i \leq n \operatorname{lcm}\left(d_{i}, d_{i}^{\prime}\right)$ divides the $p$-digits of $\frac{(q-1) r_{i}}{s_{i}}$ (this is the case when $p \equiv 1$ modulo $\left.D=\operatorname{lcm}\left(d_{i}, d_{i}^{\prime}, s_{i}\right)_{1 \leq i \leq n}\right)$, the polygons $\operatorname{GNP}(\Delta, \chi, p)$ and $\operatorname{HP}\left(\Delta, \frac{\mathbf{r}}{\mathbf{s}}, 1\right)$ coincide, and this gives a particular case of an extension of Adolphson and Sperber conjecture to twisted sums.

We end this chapter focusing on the particular case $s=2$. For a fixed odd prime number $p$, let $\chi_{2}$ denote the quadratic character, defined over $\mathbb{F}_{q}^{\times}(q$ any power of $p)$ by $\chi_{2}(x)=\omega^{\frac{q-1}{2}}(x)$. All multiplicative characters of order 2 of $\left(\mathbb{F}_{q}^{\times}\right)^{n}$ can be written as $\chi_{2}^{\varepsilon}$, with $\varepsilon=\left(\varepsilon_{1}, \ldots, \varepsilon_{n}\right) \in\{0,1\}^{n}$, and $\chi_{2}^{\varepsilon}\left(x_{1}, \ldots, x_{n}\right)=\chi_{2}^{\varepsilon_{1}}\left(x_{1}\right) \ldots \chi_{2}^{\varepsilon_{n}}\left(x_{n}\right)$. Since prime numbers strictly larger than 2 are odd, the Hodge-Stickelberger polygon actually does not depend on the residue of the prime $p$, and we denote it by $\operatorname{HS}\left(\Delta, \frac{\varepsilon}{2}\right)$. For the same reason, one can describe directly this polygon from a Poincaré series. 
Lemma 4.1. Let $\Delta$ be a convex polytope generating $\mathbb{R}^{n}$, and $\mathcal{A}_{\Delta, \frac{\varepsilon}{2}}$ the graded $\mathcal{A}_{\Delta}$-module associated to this situation. Then the polygon $\operatorname{HS}\left(\Delta, \frac{\varepsilon}{2}\right)$ is the polygon coming from the Poincaré series of $\mathcal{A}_{\Delta, \frac{\varepsilon}{2}}$.

In this case, the independence of the Hodge polygon from $p$ gives the existence of a limit.

Corollary 4.1. Let $\Delta$ be as above. When $p$ tends to infinity, the generic Newton polygon of $\Delta$ associated to the prime $p$ and the quadratic character $\chi_{2}^{\varepsilon}, \operatorname{GNP}\left(\Delta, \chi_{2}^{\varepsilon}, p\right)$, tends to the Hodge-Stickelberger polygon $\operatorname{HS}\left(\Delta, \frac{\varepsilon}{2}\right)$.

Corollary 4.2. Let $\Delta$ be as above. There is a dense open subset $\mathcal{U}$ in the space of polynomials $f(x)=\sum_{i=1}^{n} \sum_{j=-d_{i}^{\prime}}^{d_{i}} a_{i j} \mathbf{x}^{j e_{i}}$ with their coefficients in $\overline{\mathbb{Q}}$ such that for any $f$ in $\mathcal{U}$, we have

$$
\lim _{p \rightarrow+\infty} \mathrm{NP}_{q}\left(f \quad \bmod \mathfrak{p}, \chi_{2}\right)=\operatorname{HS}\left(\Delta, \frac{\varepsilon}{2}\right) .
$$

Moreover this open is defined over $\mathbb{Q}$.

\section{Polynomials associated to polytopes of exponent two.}

In this section we extend the convergence results for the generic Newton polygons to slightly more general polytopes: fix an integer $n$, and let $\left(\mathbf{f}_{1}, \ldots, \mathbf{f}_{n}\right)$ be a free family in $\mathbb{Z}^{n}$, generating a submodule $N$ contained in $2 \mathbb{Z}^{n}$. As in the preceding chapters, we choose integers $d_{1}, d_{1}^{\prime}, \ldots, d_{n}, d_{n}^{\prime}$ with $\left(d_{i}, d_{i}^{\prime}\right) \neq(0,0)$. We denote by $\Delta$ the convex polytope in $\mathbb{R}^{n}$ which is the convex hull of the set $\left\{d_{i} \mathbf{f}_{i},-d_{i}^{\prime} \mathbf{f}_{i}\right\}_{1 \leq i \leq n}$ and the origin if necessary. We shall express additive exponential sums associated to certain polynomials with Newton polytope $\Delta$ from the twisted sums studied in the former chapter; then we shall use Corollaries 4.1 and 4.2 to obtain the limit.

The aim of this section is to show the following results

Theorem 5.1. The generic Newton polygon associated to $\Delta$ and the prime $p$, $\operatorname{GNP}(\Delta, p)$, tends to the Hodge polygon $\operatorname{HP}(\Delta)$ when $p$ tends to infinity.

Theorem 5.2. There exists a dense open subset $\mathcal{U}$ defined over $\mathbb{Q}$ in the space of polynomials of the form $f(x)=\sum_{i=1}^{n} \sum_{j=-d_{i}^{\prime}}^{d_{i}} a_{i j} \mathbf{x}^{j f_{i}}$ (with their coefficients in $\overline{\mathbb{Q}}$ ) such that for any polynomial whose coefficients belong to $\mathcal{U}$, we have

$$
\lim _{p \rightarrow \infty} \mathrm{NP}_{q}(f \quad \bmod \mathfrak{p})=\operatorname{HP}(\Delta) .
$$

In the following, $p$ is an odd prime number.

Let $\mathcal{F}=\left(\mathbf{f}_{1}, \ldots, \mathbf{f}_{n}\right)$ be a free family in $\mathbb{Z}^{n}$, generating a submodule $N$ such that the quotient $\mathbb{Z}^{n} / N$ is a group with exponent 2 . We note $M=\left(f_{i j}\right)$ the base change matrix from the canonical basis to the family $\mathcal{F}$ in $\mathbf{M}_{n}(\mathbb{Z})$, and $k$ the dimension of $\mathbb{Z}^{n} / N$ as $\mathbb{F}_{2}$-vector space. We have the exact sequence

$$
0 \rightarrow \mathbb{Z}^{n} \rightarrow \mathbb{Z}^{n} \rightarrow \mathbb{Z}^{n} / N \simeq \mathbb{F}_{2}^{k} \rightarrow 0,
$$

where the first arrow is the action of $M$.

One can find a basis $\left(\mathbf{e}_{1}, \ldots, \mathbf{e}_{n}\right)$ in $\mathbb{Z}^{n}$ (as a $\mathbb{Z}$-module) such that the family $\mathbf{e}_{1}, \ldots, \mathbf{e}_{n-k}, 2 \mathbf{e}_{n-k+1}, \ldots, 2 \mathbf{e}_{n}$ is a basis for the $\mathbb{Z}$-module $N$. In other words, the matrix $M$ is equivalent, over $\mathbf{M}_{n}(\mathbb{Z})$, to the diagonal matrix whose first $n-k$ diagonal coefficients are 1 , and the last $k$ ones are 2 ; remark that $\operatorname{det} M=2^{k}$. 
Definition 5.1. We note $M_{2}$ the linear map on $\mathbb{F}_{2}^{n}$ induced by the matrix obtained from $M$ by reducing its coefficients modulo 2. Let $\bar{E}$ be its kernel, and for all $\bar{\varepsilon}=\left(\bar{\varepsilon}_{1}, \ldots, \bar{\varepsilon}_{n}\right) \in \bar{E}$, note $\varepsilon=\left(\varepsilon_{1}, \ldots, \varepsilon_{n}\right)$ the lifting of $\bar{\varepsilon}$ to $\{0,1\}^{n}$. Finally, let $E$ be the subset in $\{0,1\}^{n}$ consisting of the $\varepsilon$ when $\bar{\varepsilon}$ runs over $\bar{E}$.

We will use the set $E$ we have just defined in order to describe the integral points in a fundamental domain of $\mathbb{Z}^{n} / N$. Recall that for $\mathbf{x}=\left(x_{1}, \ldots, x_{n}\right)$, we have set ${ }^{M} \mathbf{x}=\left(y_{1}, \ldots, y_{n}\right)$, where $y_{i}=\prod_{j=1}^{n} x_{j}^{f_{j i}}$. We will also use the set $E$ to describe the image of the morphism $\varphi_{M}: \mathbf{x} \mapsto^{M} \mathbf{x}$ from $\left(k_{r}^{\times}\right)^{n}$ to itself, and to express the additive sums (as long as their $L$ functions) associated to the polynomial $f\left({ }^{M} \mathbf{x}\right)$ from twisted sums associated to $f$ and certain quadratic characters. Finally recall that we set $\chi_{2}^{\varepsilon}, \varepsilon=\left(\varepsilon_{1}, \ldots, \varepsilon_{n}\right) \in\{0,1\}^{n}$, the multiplicative character of $k^{\times n}$ defined by $\chi_{2}^{\varepsilon}(\mathbf{x})=\chi_{2}^{\varepsilon_{1}}\left(x_{1}\right) \ldots \chi_{2}^{\varepsilon_{n}}\left(x_{n}\right)$.

Lemma 5.1. $\quad$ if The set of integral points contained in the polytope

$$
\left[0,1\left[\mathbf{f}_{1} \times \cdots \times\left[0,1\left[\mathbf{f}_{n}=\left\{\sum_{i=1}^{n} x_{i} \mathbf{f}_{i}, 0 \leq x_{i}<1\right\}\right.\right.\right.\right.
$$

is $\left\{\mathbf{f}_{\boldsymbol{\varepsilon}}:=\frac{1}{2} \sum_{i=1}^{n} \varepsilon_{i} \mathbf{f}_{i}, \varepsilon \in E\right\}$.

ii) The subgroup of multiplicative characters of $\left(k_{r}^{\times}\right)^{n}$ orthogonal to the image of $\varphi_{M}$ is

$$
\left(\operatorname{Im} \varphi_{M}\right)^{\perp}=\left\{\chi_{2}^{\varepsilon}, \varepsilon \in E\right\} .
$$

Proof. The polytope $\left[0,1\left[\mathbf{f}_{1} \times \cdots \times\left[0,1\left[\mathbf{f}_{n}\right.\right.\right.\right.$ is a fundamental domain for the action of $N$ on $\mathbb{R}^{n}$ by translations. As a consequence it contains $\operatorname{det} M=2^{k}$ integral points. Since $M_{2}$ has rank $k, E$ contains $2^{k}$ elements, and it is sufficient to verify that the points $\mathbf{f}_{\varepsilon}$ are integral when $\varepsilon$ describes $E$. From its construction, the vector $\sum_{i=1}^{n} \varepsilon_{i} \mathbf{f}_{i}$ vanishes in $\mathbb{F}_{2}^{n}$, thus each coordinate of $\sum_{i=1}^{n} \varepsilon_{i} \mathbf{f}_{i}$ is even, this shows assertion $i /$.

Since we assumed $p$ odd, the group $\left(k_{r}^{\times}\right)^{n}$ is isomorphic to $\left(\mathbb{Z} /\left(q^{r}-1\right) \mathbb{Z}\right)^{n}$, with $q^{r}-1$ even. Taking the tensor product of the exact sequence (1) by the group $\mathbb{Z} /\left(q^{r}-1\right) \mathbb{Z}$, we obtain the exact sequence

$$
\left(\mathbb{Z} /\left(q^{r}-1\right) \mathbb{Z}\right)^{n} \rightarrow\left(\mathbb{Z} /\left(q^{r}-1\right) \mathbb{Z}\right)^{n} \rightarrow \mathbb{F}_{2}^{k} \rightarrow 0 .
$$

Thus the image of the morphism $\varphi_{M}$ has index $2^{k}$ in $\left(k_{r}^{\times}\right)^{n}$, and we just have to show an inclusion. One easily checks that $\chi_{2}^{\varepsilon}\left({ }^{M} \mathbf{x}\right)=\chi_{2}^{e_{1}}\left(x_{1}\right) \ldots \chi_{2}^{e_{n}}\left(x_{n}\right)$, where $e_{i}$ is the $i$-th coordinate of the vector $M \varepsilon$. As above, when $\varepsilon$ is in $E$, the vector $M \varepsilon$ has all coordinates even. Thus $\chi_{2}^{\varepsilon}$ is orthogonal to the image of $\varphi_{M}$, and this ends the proof.

We now express additive sums associated to a Laurent polynomial of the form $f\left({ }^{M} \mathbf{x}\right)$.

Proposition 5.1. Let $f \in k\left[\mathbf{x}, \mathbf{x}^{-1}\right]$ be a Laurent polynomial, and $M$ be as above; recall that ${ }^{M} f$ is the Laurent polynomial $f\left({ }^{M} x\right)$. Then we have the following decompositions

i) for the character sums

$$
\sum_{\boldsymbol{x} \in k_{r}^{\times n}} \psi\left({ }^{M} f(\mathbf{x})\right)=\sum_{\boldsymbol{\varepsilon} \in E} \sum_{\boldsymbol{x} \in k_{r}^{\times n}} \psi(f(\mathbf{x})) \chi_{2}^{\varepsilon}(\mathbf{x}) ;
$$


ii) for the $L$ function

$$
L\left({ }^{M} f, T\right)=\prod_{\varepsilon \in E} L\left(f, \chi_{2}^{\varepsilon}, T\right) .
$$

Proof. These are different avatars of Poisson's formula applied to our situation. We just show the first one: from assertion ii/ of Lemma 5.1, we have

$$
\begin{aligned}
\sum_{\mathbf{x} \in\left(k_{r}^{\times}\right)^{n}} \psi\left(f\left({ }^{M} \mathbf{x}\right)\right) & =\# \operatorname{Ker} \varphi_{M} \sum_{\mathbf{y} \in \operatorname{Im} \varphi_{M}} \psi(f(\mathbf{y})) \\
& =\# \operatorname{Ker} \varphi_{M}\left(\frac{1}{\#\left(\operatorname{Im} \varphi_{M}\right)^{\perp}} \sum_{\chi \in\left(\operatorname{Im} \varphi_{M}\right)^{\perp}} \sum_{\mathbf{x} \in\left(k_{r}^{\times}\right)^{n}} \psi(f(\mathbf{x})) \chi(\mathbf{x})\right) \\
& =\sum_{\boldsymbol{\varepsilon} \in E} \sum_{\mathbf{x} \in\left(k_{r}^{\times}\right)^{n}} \psi(f(\mathbf{x})) \chi_{2}^{\varepsilon}(\mathbf{x}),
\end{aligned}
$$

and this is exactly the desired result.

We deduce a splitting of the Newton polygon; for this we need another operation

Definition 5.2. Let $\Pi_{1}$ and $\Pi_{2}$ be two convex polygons having slopes $\left(s_{i}\right)_{1 \leq i \leq a}$ et $\left(s_{i}^{\prime}\right)_{1 \leq i \leq b}$; their concatenation is the convex polygon $\Pi_{1} \amalg \Pi_{2}$ having slopes

$$
\left(s_{i}, s_{j}^{\prime}\right)_{1 \leq i \leq a, 1 \leq j \leq b}
$$

With this definition, we deduce from Proposition 5.1 the following result

Corollary 5.1. The Newton polygon associated to the polynomial ${ }^{M} f$ is the concatenation of the Newton polygons associated to the polynomial $f$ and the characters $\chi_{2}^{\varepsilon}$ when $\varepsilon$ runs over $E$

$$
\mathrm{NP}_{q}\left({ }^{M} f\right)=\coprod_{\varepsilon \in E} \mathrm{NP}_{q}\left(f, \chi_{2}^{\varepsilon}\right)
$$

We rewrite the Hodge polygon $\operatorname{HP}(\Delta)$ from the polygons we have introduced in the sections above

Lemma 5.2. Let $\Delta_{0}$ be the convex polytope $\mathbb{R}^{n}$ which is the convex hull of the points $\left\{d_{i} \mathbf{e}_{i},-d_{i}^{\prime} \mathbf{e}_{i}\right\}_{1 \leq i \leq n}$ and the origin if necessary, for $\left(\mathbf{e}_{1}, \ldots, \mathbf{e}_{n}\right)$ a basis of the $\mathbb{Z}$-module $\mathbb{Z}^{n}$. Then the Hodge polygon associated to $\Delta$ splits in the following way

$$
\operatorname{HP}(\Delta)=\operatorname{HP}\left(\Delta_{0}\right) \coprod\left(\coprod_{\varepsilon \in E \backslash\{0, \ldots, 0\}} \operatorname{HS}\left(\Delta_{0}, \frac{\varepsilon}{2}\right)\right) .
$$

Proof. We come back to the very definition of the Hodge polygon in terms of the Poincaré series of the algebras $\mathcal{A}_{\Delta}$ and $\mathcal{A}_{\Delta_{0}, \frac{\varepsilon}{2}}$. Up to a permutation of the degrees $\left(d_{i}, d_{i}^{\prime}\right)$, and a change between $d_{i}$ and $d_{i}^{\prime}$ in some of them, we can assume that $d_{1}^{\prime}=\cdots=d_{l}^{\prime}=0$ and the $d_{i}^{\prime}$ are nonzero for $l+1 \leq i \leq n$. From Lemma 5.1, the points in $M_{\Delta}$ are the

$$
\mathbf{f}_{\mathbf{k}, \varepsilon}:=k_{1} \mathbf{f}_{1}+\cdots+k_{n} \mathbf{f}_{n}+\mathbf{f}_{\varepsilon}, \mathbf{k}=\left(k_{1}, \ldots, k_{n}\right) \in \mathbb{N}^{l} \times \mathbb{Z}^{n-l}, \varepsilon \in E,
$$

and the weight of such a point is

$$
w_{\Delta}\left(\mathbf{f}_{\mathbf{k}, \varepsilon}\right)=\sum_{i=1}^{l} \frac{k_{i}+\frac{\varepsilon_{i}}{2}}{d_{i}}+\sum_{i=l+1}^{n} \max \left(\frac{k_{i}+\frac{\varepsilon_{i}}{2}}{d_{i}},-\frac{k_{i}+\frac{\varepsilon_{i}}{2}}{d_{i}^{\prime}}\right) .
$$

We deduce, if $D$ denotes the denominator of $\Delta$, that the Poincaré series of the algebra $\mathcal{A}_{\Delta}$ can be written

$$
P_{\mathcal{A}_{\Delta}}(t)=\sum_{\boldsymbol{\varepsilon} \in E} \sum_{\mathbf{k} \in \mathbb{N}^{l} \times \mathbb{Z}^{n-l}} t^{D w_{\Delta}\left(\mathbf{f}_{\mathbf{k}, \boldsymbol{\varepsilon}}\right)} .
$$


Fix $\varepsilon \in E$. The points in $M_{\Delta_{0}, \frac{\varepsilon}{2}}$ are the $\mathbf{e}_{\mathbf{k}, \varepsilon}=k_{1} \mathbf{e}_{1}+\cdots+k_{n} \mathbf{e}_{n}+\mathbf{e}_{\boldsymbol{\varepsilon}}$, $\mathbf{k}$ running over $\mathbb{N}^{l} \times \mathbb{Z}^{n-l}$ and $\mathbf{e}_{\varepsilon}:=\frac{1}{2} \sum_{i=1}^{n} \varepsilon_{i} \mathbf{e}_{i}$, and we get

$$
w_{\Delta_{0}}\left(\mathbf{e}_{\mathbf{k}, \varepsilon}\right)=\sum_{i=1}^{l} \frac{k_{i}+\frac{\varepsilon_{i}}{2}}{d_{i}}+\sum_{i=l+1}^{n} \max \left(\frac{k_{i}+\frac{\varepsilon_{i}}{2}}{d_{i}},-\frac{k_{i}+\frac{\varepsilon_{i}}{2}}{d_{i}^{\prime}}\right) .
$$

If $D_{\varepsilon}$ is the denominator of $\left(\Delta_{0}, \frac{\varepsilon}{2}\right)$, the Poincaré series of $\mathcal{A}_{\Delta_{0}, \frac{\varepsilon}{2}}$ can be written

$$
P_{\mathcal{A}_{\Delta_{0}, \frac{\varepsilon}{2}}}(t)=\sum_{\mathbf{k} \in \mathbb{N}^{l} \times \mathbb{Z}^{n-l}} t^{D_{\boldsymbol{\varepsilon}} w_{\Delta}\left(\mathbf{f}_{\mathbf{k}, \boldsymbol{\varepsilon}}\right)}
$$

Note that $w_{\Delta_{0}}\left(M_{\Delta_{0}, \frac{\varepsilon}{2}}\right) \subset w_{\Delta}\left(M_{\Delta}\right)$, that is $D_{\varepsilon}$ divides $D$. Thus we can write

$$
P_{\mathcal{A}_{\Delta}}(t)=\sum_{\varepsilon \in E} P_{\mathcal{A}_{\Delta_{0}, \frac{\varepsilon}{2}}}\left(t^{\frac{D}{D_{\varepsilon}}}\right)
$$

and multiplying both terms by $\left(1-t^{D}\right)^{n}$, we get

$$
P_{\Delta}(t)=P_{\Delta_{0}}\left(t^{\frac{D}{D_{0}}}\right)+\sum_{\varepsilon \in E \backslash\{0, \ldots, 0\}} P_{\Delta_{0}, \frac{\varepsilon}{2}}\left(t^{\frac{D}{D_{\varepsilon}}}\right) .
$$

The result comes from the construction of the Hodge polygon of $\Delta_{0}$, and Lemma 4.1 which gives the link between $\operatorname{HS}\left(\Delta_{0}, \frac{\varepsilon}{2}\right)$ and the Poincaré series of $\mathcal{A}_{\Delta_{0}, \frac{\varepsilon}{2}}$.

From these preliminary results, we are able to show Theorems 5.1 and 5.2 .

Proof. (of Theorem 5.1) First remark that

$$
f(\mathbf{x})=\sum_{i=1}^{n} \sum_{j=-d_{i}^{\prime}}^{d_{i}} a_{i j} \mathbf{x}^{j \mathbf{f}_{i}}={ }^{M} g(\mathbf{x}),
$$

for the polynomial $g(\mathbf{x})=\sum_{i=1}^{n} \sum_{j=-d_{i}^{\prime}}^{d_{i}} a_{i j} \mathbf{x}_{i}^{j}$. From Corollary [5.1] the generic Newton polygon for the family of polynomials $f(\mathbf{x})=\sum_{i=1}^{n} \sum_{j=-d_{i}^{\prime}}^{d_{i}} a_{i j} \mathbf{x}^{j \mathbf{f}_{i}}, a_{i j} \in k$ is given by

$$
\operatorname{GNP}\left(\Delta_{0}, p\right) \coprod\left(\coprod_{\varepsilon \in E \backslash\{0, \ldots, 0\}} \operatorname{GNP}\left(\Delta_{0}, \frac{\varepsilon}{2}, p\right)\right) .
$$

Grothendieck's specialisation theorem ensures that the generic Newton polygon of the family of polynomials with Newton polytope $\Delta$ is

$$
\operatorname{GNP}\left(\Delta_{0}, p\right) \coprod\left(\coprod_{\varepsilon \in E \backslash\{0, \ldots, 0\}} \operatorname{GNP}\left(\Delta_{0}, \frac{\varepsilon}{2}, p\right)\right) \preceq \operatorname{GNP}(\Delta, p) \preceq \operatorname{HP}(\Delta) .
$$

Finally Lemma 5.2, joint with Theorem 3.1 applied to the polytope $\Delta_{0}$, and Corollary 4.1, ensure that the left-hand term tends to the right-hand one when $p$ tends to $\infty$. Thus the polygon $\operatorname{GNP}(\Delta, p)$ tends to $\operatorname{HP}(\Delta)$ when $p$ tends to $\infty$.

Proof. (of Theorem 5.2) For $f$ a polynomial such as

$$
f(\mathbf{x})=\sum_{i=1}^{n} \sum_{j=-d_{i}^{\prime}}^{d_{i}} a_{i j} \mathbf{x}^{j \mathbf{f}_{i}}
$$


with its coefficients in $\overline{\mathbb{Q}}$, we verify as above that

$$
\lim _{p \rightarrow \infty} \operatorname{NP}_{q}(f \quad \bmod \mathfrak{p})=\operatorname{HP}(\Delta)
$$

as long as $\mathcal{H}\left(a_{i j}\right) \neq 0$, where the Hasse polynomial here is

$$
\mathcal{H}\left(X_{i j}\right)=\prod_{i=1}^{n} \prod_{\varepsilon \in E} \mathcal{H}_{\left[-d_{i}^{\prime}, d_{i}\right], \frac{\varepsilon_{i}}{2}}\left(X_{i j}\right)
$$

\section{REFERENCES}

[1] A. Adolphson, S. Sperber : Exponential sums and Newton polyhedra: Cohomology and estimates, Ann. Math 130 (1989), 367-408.

[2] A. Adolphson, S. Sperber: On twisted exponential sums, Math. Ann. 290 (1991), 713-726.

[3] A. Adolphson, S. Sperber : Twisted exponential sums and Newton polyhedra, J. reine angew. Math. 443 (1993), 151-177.

[4] R. Blache, É. FÉRARD : Newton stratification for polynomials: the open stratum, J. Number Th. 123 (2007), 456-472.

[5] R. Blache, É. FÉrard, H.J.Zhu : Hodge-Stickelberger polygons for L-functions of exponential sums of $P\left(x^{s}\right)$, Math. Res. Letters 15 (2008), 1053-1072.

[6] P. Deligne : La conjecture de Weil : I. Publ. Math. IHES 43 (1974), 273-307.

[7] J. Denef, F. Loeser : Weights of exponential sums, intersection cohomology, and Newton polyhedra. Inv. Math. 106 (1991), 275-294.

[8] B. Dwork: On the zeta function of a hypersurface. Publ. Math. IHES 12 (1962), 5-68.

[9] R. Garcia Lopez : A note on $L$-series and Hodge spectrum of polynomials. El. Res. Ann. Math. Sc. 16 (2009), 56-62.

[10] A. Grothendieck : Formule de Lefschetz et rationalité des fonctions $L$, Séminaire Bourbaki, exposé $279,1964 / 65$.

[11] M. Henk, J. Richter-Gebert, G. Ziegler : Basic properties of convex polytopes, in Handbook of Discrete and Computational Geometry, CRC Press, 1997.

[12] N.M. KaTz : Slope filtration of F-crystals, Astérisque 63 (1979), 113-164.

[13] N. Koblitz: $p$-adic numbers, $p$-adic analysis, and zeta-functions, (Second edition), Graduate Texts in Mathematics 58. Springer-Verlag, New York, 1984.

[14] A.G. Kouchnirenko : Polyèdres de Newton et nombres de Milnor. Inv. Math. 32 (1976), $1-31$.

[15] H. LI, H. J. ZHU : Zeta functions of totally ramified $p$-covers of the projective line. Rend. Sem. Mat. Univ. Padova, 113 (2005), 203-225.

[16] C. LiU : Generic exponential sums associated to Laurent polynomials in one variable, Preprint avalaible at http://arxiv.org/abs/0802.0271 (2008).

[17] P. RobBa : Index of $p$-adic differential operators III. Applications to twisted exponential sums. Astérisque, 119-120 (1984), 191-266.

[18] D. WAN : Newton polygons for zeta and L-functions, Ann. Math. 137 (1993), 249-296.

[19] D. WAN : Variation of $p$-adic Newton polygons for $L$-functions of exponential sums, Asian J. Math. 8 (2004), 427-472.

[20] H. J. ZHU: $p$-adic variation of $L$ functions of one variable exponential sums, I. Amer. J. Math. 125 (2003).

[21] H. J. ZHU : Asymptotic variation of $L$-functions of one-variable exponential sums. J. Reine Angew. Math. 572 (2004), 219-233.

EQuipe LAMIA, IUFM DE LA GuAdELOUPE

E-mail address: rblache@iufm.univ-ag.fr 